\title{
Limits of Infinite Interaction Radius, Dimensionality and the Number of Components for Random Operators with Off-Diagonal Randomness
}

\author{
A. M. Khorunzhy and L. A. Pastur \\ Institute for Low Temperature Physics, Academy of Sciences of Ukraine, 47 Lenin Ave, Kharkiv, \\ 310164, Ukraine
}

Received September 15, 1992

\begin{abstract}
We consider random one-body operators that are analogs of the statistical mechanics Hamiltonians with a varying interaction radius $R$, the dimensionality of space $d$ and the number of the field components (orbitals) $n$. We prove that all the moments of the Green functions for nonreal energies of these operators converge as $R, d, n \rightarrow \infty$ to the products of the average Green functions, just as in the mean field approximation of statistical mechanics. We find in particular the selfconsistent equation for the limiting integrated density of states and the limiting form of the conductivity, which is nonzero on the whole support of the integrated density of states.
\end{abstract}

\section{Introduction}

The spectral and related properties of random operators have attracted considerable interest in both physical and mathematical literature. It is believed, in particular, that under suitable conditions the spectrum of these operators is pure point and dense. This has been proven under various circumstances (in the one-dimensional case or in any dimension near the edges of the spectrum or for a sufficiently large random potential). Therefore, although many important problems still remain open here (twodimensional localization, calculation of the low frequency conductivity and other physical quantities, etc.), the strong disorder (or low energy) regime in the spectral theory of random operators can be regarded as rather well understood rigorously, especially in comparison with the weak disorder (or high energy) regime. This regime is almost unexplored rigorously despite extensive numerical and theoretical physics studies. In particular, the weak localization theory (see e.g. review [19]) allows us to calculate the so-called quantum corrections for many important physical quantities and, being supplemented by some renormalization group ideas, predicts complete localization in one and two dimensions, a mixed spectrum in higher dimensions and, as a result, the metal-insulator transition. The latter is largely similar to phase transition in statistical mechanics. Thus, based on the statistical mechanics experience it is natural 
to try to develop some versions of the selfconsistent approaches that are widely accepted tools of study of difficult phase transition problems. In statistical mechanics the most widely accepted selfconsistent schemes, such as numerous versions of the molecular field approximation or the spherical model, can be obtained as the limits of the infinite interaction radius, dimensionality of space or the number of field components (dimensionality of the spin space). An important feature of these limits is that they are nonperturbative in the sense that the interaction responsible for the phase transition is not assumed to be small in corresponding models. Small (or at least suppressed) in these limits are the fluctuations of the order parameter and other important physical quantities.

In this paper we study some random operators that can be regarded as analogs of statistical mechanics models with a large interaction radius $R$, a large dimensionality of space $d$ or a large number of components $n$. We calculate the integrated density of states (IDS) of these operators and the conductivity in the limits of infinite $R, d$ and $n$. In fact, the latter model was introduced and studied at the physical level of rigour by Wegner [31] (for some rigorous results on the IDS of this model see also [7]).

We use the method which is analogous to the method of correlation equations (or cluster expansion) of statistical mechanics and allows us to calculate the IDS and the conductivity (more exactly, the measure which is naturally associated with the conductivity) in all three limits.

In principle, our method can also be used to construct the respective $R^{-1}-, d^{-1}$ and $n^{-1}$-expansions for which our limit expressions are the leading terms. We hope to discuss these expansions in subsequent publications (see however [33] where the physical $n^{-1}$-expansion scheme was developed for the conductivity and [7] for rigorous expansion for the IDS).

The paper is organized as follows. In Sect. 2 we introduce the models and formulate the main results, according to which the IDS and the conductivity are practically the same for all three models and can be calculated from some selfconsistent equation. The former fact should be contrasted with statistical mechanics, where the limits $R=\infty$ and $d=\infty$ coincide with the mean field approximation, while the limit $n=\infty$ coincides with the spherical model. In Sect. 3 we derive infinite systems of equations for the moments of the Green functions of the respective operators that are our main technical tools. In Sect. 4 we solve these equations in the limits $R, d$, $n=\infty$, derive a selfconsistent equation for the limiting IDS (see Eq. (2.15) below) and in Sect. 5 we study some properties of the IDS (existence of the bounded density, location of the support, the form of singularities at the edges of the support). In Sect. 6 we calculate the conductivity of the respective disordered system in the same limits. Section 7 is devoted to discussion of our results, in particular their relation to the random matrix theory and their possible interpretations.

\section{Models and Results}

We start from the random operator containing an analog of the interaction radius. Let $H_{R}$ be the selfadjoint operator, acting in $\ell^{2}\left(\mathbb{Z}^{d}\right)$ and defined by the matrix

$$
H_{R}(x, y)=h(x-y)+R^{-d / 2} \phi((x-y) / R) W(x, y) .
$$

Here $x, y \in \mathbb{Z}^{d}$,

$$
h(-x)=h^{*}(x), \quad \sum_{x \in \mathbb{Z}^{d}}|h(x)|<\infty,
$$


$R>0, \phi(t), t \in \mathbb{R}^{d}$ is a piece-wise continuous, nonnegative and such that

$$
|\phi(t)| \leq \phi_{0}<\infty, \quad \phi(t)=0,|t|>1, \int_{\mathbb{R}^{d}} \phi^{2}(t) d t=1,
$$

and

$$
W(x, y)=W(y, x)
$$

are identically distributed and independent (modulo the above symmetry condition) random variables such that for all $x, y \in \mathbb{Z}^{d}$,

$$
\begin{aligned}
\mathbb{E}\{W(x, y)\}= & 0 \\
\mathbb{E}\left\{W\left(x_{1}, y_{1}\right) W\left(x_{2}, y_{2}\right)\right\}= & w^{2}\left\{\delta\left(x_{1}-x_{2}\right) \delta\left(y_{1}-y_{2}\right)\right. \\
& \left.+\delta\left(x_{1}-y_{2}\right) \delta\left(y_{1}-x_{2}\right)\right\} .
\end{aligned}
$$

Our second random operator $H_{d}$ contains explicitly the dimensionality $d$ of the space $\mathbb{Z}^{d}$. It acts also in $\ell^{2}\left(\mathbb{Z}^{d}\right)$ and is defined by the matrix

$$
H_{d}(x, y)=h_{d}(x-y)+(2 d)^{-1 / 2} W_{1}(x, y) \text {. }
$$

where

$$
H_{d}(x)=d^{-1 / 2} \sum_{j=1}^{d} h_{1}\left(x_{j}\right) \prod_{k \neq j} \delta\left(x_{k}\right), \quad h_{1}(0)=0,
$$

$\delta(x)$ is the Kronecker symbol and $h_{1}(x), x \in \mathbb{Z}^{1}$, satisfies (2.1b),

$$
W_{1}(x, y)= \begin{cases}W(x, y), & |x-y|=1 \\ 0, & |x-y| \neq 1\end{cases}
$$

and $W(x, y)$ are as in (2.1d)-(2.1f).

The simplest and quite important example of the operator $h_{d}$ is the discrete Laplacian for which

$$
h_{1}(x)=0, \quad|x| \neq 1 .
$$

We will see later that the form (2.2b) of the unperturbed operator $h_{d}$ in $(2.2 \mathrm{a})$, which is more special than $(2.1 \mathrm{~b})$, is dictated by the requirement to have the nontrivial $d=\infty$ limit for the IDS. We note that the $d=\infty$ limit is technically most complicated to derive among the three considered.

The third operator $H_{n}$ acts in $\ell^{2}\left(\mathbb{Z}^{d}\right) \otimes \mathbb{C}^{n}$ and is defined by the matrix

$$
H(\alpha, x ; \beta, y)=h(x-y) \delta_{\alpha \beta}+n^{-1 / 2} \delta(x-y) W_{\alpha \beta}(x),
$$

where $x, y \in \mathbb{Z}^{d}, \alpha, \beta=1, \ldots, n, h(x)$ is the same as in $(2.1), \delta(x)$ is the Kronecker symbol in $\mathbb{Z}^{d}$,

$$
W_{\alpha \beta}(x)=W_{\beta \alpha}(x)
$$

and $W_{\alpha \beta}(x)$ are identically distributed and independent for $1 \leq \alpha \leq \beta \leq n$ random variables such that (cf. (2.1e,f)) for all $x \in \mathbb{Z}^{d}$ and $1 \leq \alpha \leq \beta \leq n$,

$$
\begin{aligned}
\mathbb{E}\left\{W_{\alpha \beta}(x)\right\} & =0, \\
\mathbb{E}\left\{W_{\alpha_{1} \beta_{1}}\left(x_{1}\right) W_{\alpha_{2} \beta_{2}}\left(x_{2}\right)\right\} & =w^{2} \delta\left(x_{1}-x_{2}\right)\left\{\delta_{\alpha_{1} \alpha_{2}} \delta_{\beta_{1} \beta_{2}}+\delta_{\alpha_{1} \beta_{2}} \delta_{\alpha_{2} \beta_{1}}\right\} .
\end{aligned}
$$

The random operator (2.3) is a special case of the operator introduced by Wegner [31] (the case of the site-diagonal disorder, according to Wegner's terminology). It 
can be regarded as the $n$-component analog of the discrete Schrödinger operator (the Anderson model) or as the Hamiltonian of a disordered system in the dimensions $d+d_{1}, d_{1}=n$, in which the random potential in $n$ "transverse" dimensions is considered in the "mean field" approximation.

In all three cases we consider "maximally random" operators with independent (apart from the Hermitian symmetry) entries (see however the end of this section, in particular, Eq. (2.30)). Note also that in (2.1f) and (2.3d) we assume that the variance of the diagonal random entries is twice large as that of the off-diagonal ones. This assumption is very convenient and simplifies considerably many of our formulae. On the other hand our main results are independent of the diagonal entries and coincide with those for the zero diagonal entries. We demonstrate this in the Appendix.

All three families of random matrices (2.1)-(2.3) define the essentially selfadjoint metrically transitive operators in the sense of [25]. They have the form of a nonrandom translationally invariant part and a fluctuating random part explicitly containing the parameters $R, d$ or $n$ that we are going to send to infinity. The random parts are such that the larger these parameters are, the more "extended" and smaller randomness is. Similar scaling of the interaction is widely used in the mean field and the spherical approximations of statistical mechanics (see [3,12, 15, 20, 26-28]).

Our intention is to study, first of all, the simplest, though rather important in several respects, spectral characteristic of random operators known as the integrated density of states (IDS). It is defined as the measure

$$
N_{a}(d \lambda)=\mathbb{E}\left\{\mathscr{E}_{a}(0,0 ; d \lambda)\right\}
$$

where $a$ is $R$ or $d$ and $\mathscr{E}_{a}(d \lambda)$ is the resolution of identity of the operators $H_{a}, a=R$, $d$, and $\mathscr{E}_{a}(x, y ; d \lambda), x, y \in \mathbb{Z}^{d}$ are the respective matrices. For the model (2.3),

$$
N_{n}(d \lambda)=\mathbb{E}\left\{n^{-1} \sum_{\alpha=1}^{n} \mathscr{E}_{n}(\alpha, 0 ; \alpha, 0 ; d \lambda)\right\},
$$

where $\mathscr{E}_{n}(\alpha, x ; \beta, y ; d \lambda)$ is the matrix of the resolution of identity of the operator $H_{n}$. For another definition of the IDS, which is based on a kind of the thermodynamic limiting transition, and for equivalence of definitions, see [25].

The natural condition of there being nonzero and noninfinite IDS in these limits fixes the normalization factors $R^{-1 / 2}, d^{-1 / 2}$ and $n^{-1 / 2}$ in $(2.1)-(2.3)$. In full extent this will be seen below. Here we give simple arguments. Indeed, according to (2.4) and the spectral theorem for $a=R, d$,

$$
\int_{-\infty}^{\infty} \lambda^{2} N_{a}(d \lambda)=\mathbb{E}\left\{\left(H_{a}^{2}\right)(0,0)\right\}
$$

and for $a=n$,

$$
\int_{-\infty}^{\infty} \lambda^{2} N_{n}(d \lambda)=\mathbb{E}\left\{n^{-1} \sum_{\alpha=1}^{n}\left(H_{n}^{2}\right)(\alpha, 0 ; \alpha, 0)\right\} .
$$

Now, calculating the r.h.s. of these relations from (2.1)-(2.3), we find that it is nonzero and finite in the limits $R, d, n \rightarrow \infty$. 
For an arbitrary nonnegative measure $\mu(d t)$ on $\mathbb{R}$, such that

$$
\int_{\mathbb{R}} \mu(d t)=1
$$

we define its Stieltjes transform

$$
f(z)=\int_{-\infty}^{\infty}(t-z)^{-1} \mu(d t), \quad \operatorname{Im} z \neq 0 .
$$

It is an analytic function for $\operatorname{Im} z \neq 0$, having asymptotics

$$
f(z)=-z^{-1}+o\left(z^{-1}\right), \quad z \rightarrow \infty,
$$

and such that

$$
\operatorname{Im} f(z) \cdot \operatorname{Im} z>0, \quad \operatorname{Im} z \neq 0 .
$$

The Stieltjes transform uniquely determines the respective measure, since due to the Stieltjes-Perron inversion formula for any interval $\Delta=\left(\lambda_{1}, \lambda_{2}\right)$ whose endpoints are continuity points of $\mu(d t)$,

$$
\mu(\Delta)=\lim _{\eta \downarrow 0}(2 \pi i)^{-1} \int_{\lambda_{1}}^{\lambda^{2}}[f(\lambda+i \eta)-f(\lambda-i \eta)] d \lambda .
$$

Denote by $N_{0}(d \lambda)$ the IDS of the unperturbed operators in (2.1)-(2.3). It is easy to show that for these Toeplitz operators,

$$
N_{0}(d \lambda)=\operatorname{mes}\left\{\tilde{h}(k) \in d \lambda, k \in \mathbb{T}^{d}\right\},
$$

where $\mathbb{T}^{d}=[0,1]^{d}$ is $d$-dimensional torus and

$$
\tilde{h}(k)=\sum_{x \in \mathbb{Z}^{d}} h(x) \exp (2 \pi i k x)
$$

is the symbol of these operators.

Our result for the IDS is (see Theorem 1 below) that the measures $N_{a}(d \lambda), a=$ $R, d, n$ converge weakly as $a \rightarrow \infty$ to the limit $N(d \lambda)$ whose Stieltjes transform

$$
r(z)=\int(\lambda-z)^{-1} N(d \lambda)
$$

can be found as a unique solution in the class (2.10) of the equation

$$
r(z)=r_{0}\left(z+w^{2} r(z)\right)
$$

in which $r_{0}(z)$ is the Stieltjes transform of the IDS (2.12) of the unperturbed (nonrandom) operators in (2.1)-(2.3).

Note that for the operator $H_{d}$ of (2.2) the unperturbed operator and its IDS depend also on the parameter $d$. Therefore, unlike $H_{R}$ and $H_{n}$, in the case of $H_{d}$ the limiting transition $d \rightarrow \infty$ affects $N_{0}(d \lambda)$ also. More precisely, in this case $N_{0}(d \lambda)$ is given by the limit of (2.2b), (2.12) and (2.13) and is

$$
N_{0}(d \lambda)=\left(2 \pi h_{2}\right)^{-1 / 2} \exp \left(-\lambda^{2} / 2 h_{2}\right) d \lambda,
$$


where

$$
h_{2}=\sum_{x \in \mathbb{Z}} h_{1}^{2}(x)
$$

Equation (2.15) was found by Wegner for the operator (2.3) in the limit $n=$ $\infty$ (infinite number of the orbitals in the terminology of Wegner). In fact, this equation had appeared before Wegner's paper as the equation for the limit eigenvalue distribution of some $n \times n$ random matrices in the limit $n=\infty$ [24]. This distribution is known as the deformed semicircle law. The situation here is similar to that in statistical mechanics, where selfconsistent equations of the Curie-Weiss model (the mean field approximation) and the spherical model had been proposed long before it was understood that these are the limits of an infinite interaction radius [20] and an infinite number of components $[15,28]$ of the classical Heisenberg ( $n$-vector) model.

Note also that the Wegner model (2.3) seems to be the closest to the model with pure diagonal randomness, i.e. to the discrete Schrödinger operator with a random potential. Nevertheless it has the same limiting IDS as the seemingly much more "off-diagonal" disorder models (2.1) and (2.2).

Now we shall outline the strategy of derivation of (2.15). Consider the family $\mathbb{E}\left\{\prod_{i=1}^{k} G\left(x_{i}, y_{i}\right)\right\}, k \geq 1$, of the moments of the Green function of the random operators (2.1)-(2.3). By using the resolvent identity, we derive for this family an infinite system of linear relations (that can be regarded as analogs of the BBGKY or the Kirkwood-Salzburg equations in statistical mechanics). Some terms in these relations contain the small parameter $a^{-1}$ in front of them. Treating these relations as an infinite system of equations for the moments and neglecting the small terms, we observe that the truncated system admits the factorized solution $\prod_{i=1}^{k} \mathscr{G}\left(x_{i}-y_{i}\right)$, $k \geq 1$, where $\mathscr{G}(x-y)$ is the Green function of the Toeplitz operator which is the sum of the nonrandom part of $H_{a}$ and the effective coordinate-independent potential $w^{2} \delta(x-y) \mathscr{G}(0)$. This yields Eq. (2.15) which is in this scheme the solvability condition for the truncated system.

To justify this procedure, we act again as in statistical mechanics. Namely, we consider our infinite system as a linear equation in some Banach space containing, in particular, our family of moments. We prove that if the imaginary part of the energy is large enough, then the nonsmall part of the equation defines the contracting linear operator and that the norm of the remainder is small.

Thus, our central technical result (see Theorem 4.1) is that in the limit $a=\infty$ the moments of the Green function of our random operators (2.1)-(2.3) are factorized into the products of the first moments, and these first moments are to be found selfconsistently, by solving the nonlinear functional equation (2.15). This result is fairly similar to the main technical result of the mean field approximation $(R, d=\infty$ limit) and the spherical limit $(n=\infty$ limit) in statistical mechanics, where the correlation functions of all orders are factorized into products of the correlation function of order one (mean field approximation) or of orders one and two (spherical model). We refer to the papers $[12,13]$ for some form of the latter results and for references.

Consider now the conductivity of a disordered system, described by the Hamiltonians (2.1)-(2.3). According to the Kubo formula, the conductivity of $d$-dimensional ideal Fermi gas at a temperature $T$, described by a one-body Hamiltonian $H$ and 
subjected to an external alternating electric field of the frequency $\nu$, is [21]

$$
\sigma_{\mathrm{ac}}(\nu, T)=2 e^{2} \pi^{-1} \int_{-\infty}^{\infty} \nu^{-1}\left[n_{F}(E+\nu)-n_{F}(E)\right] \sigma(\nu, E) d E .
$$

Here $e$ is the electron charge, $n_{F}(E)=\left(\exp \left\{\left(E-E_{F}\right) / T\right\}+1\right)^{-1}$ is the Fermi distribution function, $E_{F}$ is the Fermi energy,

$$
\sigma(\nu, E)=m(E+\nu, E),
$$

and $m\left(\lambda_{1}, \lambda_{2}\right)$ is the density of the measure

$$
M\left(d \lambda_{1}, d \lambda_{2}\right)=\sum_{k=1}^{d} \mathbb{E}\left\{\left[\hat{v}_{k} \mathscr{E}\left(d \lambda_{1}\right) \hat{v}_{k} \mathscr{E}\left(d \lambda_{2}\right)\right](0,0)\right\}
$$

on $\mathbb{R}^{2}$ in which

$$
\hat{v}=i[H, \hat{x}]
$$

is the velocity operator, $\hat{x}=\left(x_{1}, \ldots, x_{d}\right)$ is the coordinate operator and $\mathscr{E}(d \lambda)$ is the resolution of identity of the Hamiltonian $H$.

For $T=0,(2.17)$ has the form

$$
\sigma_{\mathrm{ac}}(\nu, 0)=\nu^{-1} \int_{E_{F}}^{E_{F}+\nu} \sigma(\nu, E) d E
$$

and for low frequencies $\nu \ll E_{F}$,

$$
\sigma_{a c}(\nu, 0)=2 e^{2} \pi^{-1} m\left(E_{F}+\nu, E_{F}\right)(1+o(1)), \quad \nu \rightarrow 0,
$$

i.e. the low frequency conductivity (dc conductivity in particular) can be expressed through the density $m\left(\lambda_{1}, \lambda_{2}\right)$ itself.

Consider first the case of $a=R$. According to (2.19), (2.20) and (2.1),

$$
M\left(\mathbb{R}^{2}\right)=\sum_{x} x^{2}\left(|h(x)|^{2}+w^{2} R^{-d} \phi^{2}(x / R)\right) .
$$

Thus, to obtain a finite and nontrivial answer in the limit $R=\infty$, we have to consider the normalized measure

$$
M^{(R)}\left(d \lambda_{1}, d \lambda_{2}\right)=R^{-2} M\left(d \lambda_{1}, d \lambda_{2}\right),
$$

and with this normalization we can set without loss of generality the nonrandom part of $(2.1)$ to be zero $(h(x) \equiv 0)$.

Similar arguments show that for $a=n, d$ the properly normalized measures are

$$
M^{(d)}\left(d \lambda_{1}, d \lambda_{2}\right)=M\left(d \lambda_{1}, d \lambda_{2}\right)
$$

and (cf. (2.5))

$$
M^{(n)}\left(d \lambda_{1}, d \lambda_{2}\right)=n^{-1} \sum_{\alpha=1}^{n} \sum_{j=1}^{d} \mathbb{E}\left\{\left[\hat{v}_{j} \mathscr{E}^{(n)}\left(d \lambda_{1}\right) \hat{v}_{j} \mathscr{E}^{(n)}\left(d \lambda_{2}\right)\right](\alpha, 0 ; \alpha, 0)\right\},
$$

respectively. 
One more reason to introduce the normalized sum $n^{-1} \sum_{\alpha=1}^{n}$ in the definitions of the IDS (2.5) and the conductivity (2.26) corresponding to the hamiltonian (2.3) is that both these quantities are thermodynamic limits of the corresponding quantities for the finite system confined to the box $\Lambda \subset \mathbb{Z}^{d}$. Namely, if $H_{\Lambda}$ is the Hamiltonian of a finite system, i.e. the restriction of (2.3) to $\Lambda$, then

$$
\begin{aligned}
N_{\Lambda}^{(n)}(d \lambda) & =(n|\Lambda|)^{-1} \operatorname{Tr} \mathscr{E}_{\Lambda}^{(n)}(d \lambda) \\
M_{\Lambda}^{(n)}\left(d \lambda_{1}, d \lambda_{2}\right) & =(n|\Lambda|)^{-1} \operatorname{Tr} \sum_{j=1}^{d} \hat{v}_{j} \mathscr{E}_{\Lambda}^{(n)}\left(d \lambda_{1}\right) \hat{v}_{j} \mathscr{E}_{\Lambda}^{(n)}\left(d \lambda_{2}\right) .
\end{aligned}
$$

The presence of the traces in these formulas follows from the general principles of quantum statistical mechanics and the normalization factor $(n|\Lambda|)^{-1}$ is just the inverse total number of the degrees of freedom. Thus, (2.27) and (2.28) are the quantities per one degree of freedom. It can be shown that after performing the thermodynamic limiting transition $\Lambda \rightarrow \infty$, (2.27) and (2.28) take the forms (2.5) and (2.26), respectively. For the IDS the corresponding proof can be found in [25], for the conductivity it can be obtained by similar arguments.

According to (2.17)-(2.22), the conductivity is determined by the densities of the measures (2.24)-(2.26). The existence of bounded densities of these measures in the generality which we need here has not been proved. We will not discuss here this important general question of mathematical physics of disordered systems and will not study the convergence of these densities. Rather, we will consider the weak convergence of measures (2.24)-(2.26).

Since calculation of the weak limit of measures is equivalent to calculation of their Stieltjes transforms for a nonzero imaginary part of the energy (spectral variable), we can regard this imaginary part as an analogue of a nonzero symmetry breaking field amplitude in statistical mechanics models. Symmetry breaking field is widely used in statistical mechanics calculations of various physical quantities, their infinite $R, d$ and $n$ limits in particular. Thus, our procedure of calculation of the same limits of the density of states and the conductivity via calculation of the respective limits of their Stieltjes transforms can be regarded as application of an analogue of this statistical mechanics technique. By using this procedure, we prove in Sect. 6 that the measures $M^{(a)}\left(d \lambda_{1}, d \lambda_{2}\right)$ converge weakly as $a \rightarrow \infty$ to the limiting measures with the densities

$$
\begin{aligned}
& m\left(\lambda_{1}, \lambda_{2}\right)=\varrho\left(\lambda_{1}\right) \varrho\left(\lambda_{2}\right) \\
& \quad \times \begin{cases}w^{2} \int_{\mathbb{R}^{d}}|x|^{2} \phi^{2}(x) d x, & a=R, \\
w^{2}, & a=d, \\
w^{4} \int_{\mathbb{T}^{d}} \frac{|\nabla \tilde{h}(k)|^{2} d k}{\left|\tilde{h}(k)-\zeta\left(\lambda_{1}+i 0\right)\right|^{2}\left|\tilde{h}(k)-\zeta\left(\lambda_{2}+i 0\right)\right|^{2}}, & a=n,\end{cases}
\end{aligned}
$$

where $\zeta(z)=z+w^{2} r(z)$. Results (2.29a) and (2.29b) are obtained for the zero translational-invariant parts of (2.1) and (2.2). According to (2.23) and (2.24) in the former case this part does not contribute to the conductivity, while in the latter case this assumption simplifies considerably the calculations. The result $(2.29 \mathrm{c})$ was obtained by Wegner [31] by perturbation theory arguments. 
We discuss these formulae in Sect. 7. Here we mention three more variants of our results.

In the first variant we consider correlated entries in the random part of (2.1)-(2.3) restricting ourselves for definiteness to the case of $a=R$. Assume that $W(x, y)$ in (2.1) are Gaussian-distributed random variables which satisfy (2.1e) and

$$
\mathbb{E}\left\{W\left(x_{1}, y_{1}\right) W\left(x_{2}, y_{2}\right)\right\}=B\left(x_{1}-x_{2}, y_{1}-y_{2}\right)+B\left(x_{1}-y_{2}, y_{1}-x_{2}\right)
$$

instead of (2.1f). Here $B(x, y)$ is a positive definite function on $\mathbb{Z}^{d} \times \mathbb{Z}^{d}$ such that $B(x, y)=B(y, x)=B(-x, y)$ and

$$
\sum_{x, y \in \mathbb{Z}^{d}}|B(x, y)|<\infty .
$$

In other words, now we introduce the statistical correlations of each $W(x, y)$ with $W$ 's in its neighbourhood and in the neighbourhood symmetrical with respect to the principal diagonal. However we restrict ourselves to the Gaussian-distributed $W$ 's.

By the same approach based on the moment equations, it can be shown that in this case [16]

$$
r(z)=\int_{\mathbb{T}^{d}} r(p, z) d p,
$$

and $r(p, z)$ is the unique solution of the equations

$$
\begin{aligned}
& r(p, z)=[\tilde{h}(p, z)-z-\Delta(p, z)]^{-1} \\
& \Delta(p, z)=\int_{\mathbb{T}^{d}} \tilde{B}(p, q) r(q, z) d q
\end{aligned}
$$

in which $\tilde{h}(p)$ and $\tilde{B}(p, q)$ are the Fourier transforms of $h(x)$ and $B(x, y)$ given by (2.30), respectively. Besides, the solution of this equation should be sought in the class of functions continuous in $p \in \mathbb{T}^{d}$ for each $z, \operatorname{Im} z \neq 0$, analytic in $z, \operatorname{Im} z \neq 0$ for each $p \in \mathbb{T}^{d}$ and such that for any $\psi(p) \in \mathscr{L}^{2}\left(\mathbb{T}^{d}\right)$ with unit norm we have

$$
\begin{gathered}
\operatorname{Im}\left[\int_{\mathbb{T}^{d}} r(p, z)|\psi(p)|^{2} d p\right] \cdot \operatorname{Im} z>0, \quad \operatorname{Im} z \neq 0 \\
\left.\quad \sup _{\psi} \sup _{\eta \geq 1} \eta\left|\int_{\mathbb{T}^{d}} r(p, i \eta)\right| \psi(p)\right|^{2} d p \mid \leq 1 .
\end{gathered}
$$

The result of this type, including Eqs. (2.32)-(2.33) was first obtained by Wegner [31] for the case of $a=n$ by perturbation theory arguments.

In the second variant of the above results we replace the translational-invariant "unperturbed" part in (2.1)-(2.3) by a diagonal one. More precisely, restricting ourselves again to the case of $a=R$, we assume that the Toeplitz operator $h(x-y)$ in (2.1) is replaced by the diagonal operator $\delta(x-y) h(x)$, where now $h(x), x \in \mathbb{Z}^{d}$ is an ergodic field independent of all $W$ 's. Thus, we consider the random operator

$$
\delta(x-y) h(x)+R^{-d / 2} \phi((x-y) / R) W(x, y)
$$

instead of (2.1). This operator is again the metrically transitive operator in the sense of [25] and admits the IDS. If $F(d h)=P\{h(x) \in d h\}$ is the probability distribution 
of $h(x)$ (which coincides with the IDS of the diagonal part of (2.34)), then the $R=\infty$ limit of the IDS of (2.34) exists and can be found from the same Eqs. (2.14)-(2.15) in which the role of $N_{0}(d \lambda)$ is played by $F(d \lambda)$.

In the third variant we consider Hermitian random matrices instead of symmetric ones with real-valued entries. In this case (and for $a=R$ again) $W(x, y), x, y \in \mathbb{Z}^{d}$, are complex-valued random variables with the zero mean value and such that $\overline{W(x, y)}=W(x, y), W\left(x_{1}, y_{1}\right)$ is independent of $W\left(x_{2}, y_{2}\right)$ for all pairs $\left(x_{2}, y_{2}\right) \neq$ $\left(x_{1}, y_{1}\right),\left(y_{1}, x_{2}\right)$ and

$$
\mathbb{E}\left\{W^{2}(x, y)\right\}=0, \mathbb{E}\left\{|W(x, y)|^{2}\right\}=2 w^{2} .
$$

We can set $W(x, y)=W_{1}(x, y)+i W_{2}(x, y)$, where $W_{j}(x, y), j=1,2$, are two independent copies of real-valued random variables entering into (2.1). Our results are also valid in this case (with $w^{2}$ replaced by $2 w^{2}$ ). The method of proof is here also based on the technique of the moment equations which was used in all previous cases.

\section{Equations for the Green Function Moments}

Since derivation of the infinite system of the linear relations for the moments of the Green functions is rather cumbersome, we divide the problem into two parts. First, we will derive these relations for the operators (2.1)-(2.3) with the Gaussian-distributed random variables $W$ 's. After that we will give the derivation for arbitrarily distributed $W$ 's with finite third moment. However, for $a=d$ we will restrict ourselves to the case of $h(x) \equiv 0$ in (2.3a). Derivation of the respective relations for the nonzero nonrandom part in (2.3) will be published elsewhere.

Definition. Let $f\left(X_{k} ; Y_{k}\right)$ be a complex-valued function of $X_{k}=\left(x_{1}, \ldots, x_{k}\right), Y_{k}=$ $\left(y_{1}, \ldots, y_{k}\right), x_{i}, y_{\imath} \in \mathbb{Z}^{d}, i=1, \ldots, k, k \geq 1$. Then,

$$
\|f\|_{k}^{(a)}= \begin{cases}\sup _{X_{k}, Y_{k}}\left|f\left(X_{k} ; Y_{k}\right)\right|, & a=R, n \\ {\left[\sup _{y_{1}} \sum_{x_{1}} \ldots \sup _{y_{k}} \sum_{x_{k}}\left|f\left(X_{k} ; Y_{k}\right)\right|^{2}\right]^{1 / 2},} & a=d .\end{cases}
$$

Proposition 3.1. Let $H_{a}, a=R, d, n$ be the random operators, defined by (2.1)-(2.3) with the Gaussian-distributed random variables $W$ 's,

$$
G(x, y ; z)= \begin{cases}\left(H_{a}-z\right)^{-1}(x, y), & a=R, d \\ n^{-1} \sum_{\alpha=1}^{n}\left(H_{n}-z\right)^{-1}(\alpha, x ; \alpha, y), & a=n\end{cases}
$$

$\eta=|\operatorname{Im} z| \neq 0$, and

$$
g(x-y)=(h-z)^{-1}(x, y)
$$

be the Green function of the Toeplitz operator $h$, defined by the nonrandom part in (2.1)-(2.3). Introduce the moments

$$
F_{k}\left(X_{k} ; Y_{k}\right)=\mathbb{E}\left\{\prod_{i=1}^{k} G\left(x_{i}, y_{i}\right)\right\}
$$


Then,

$$
\begin{aligned}
F_{1}\left(x_{1} ; y_{1}\right)= & g\left(x_{1}-y_{1}\right)+w^{2} \sum_{s, t} \chi_{a}(s-t) F_{2}\left(x_{1}, s ; t, s\right) g\left(t-y_{1}\right) \\
& +S_{1}^{(a)}\left(x_{1} ; y_{1}\right), \\
F_{k}\left(X_{k} ; Y_{k}\right)= & F_{k-1}\left(X_{k-1} ; Y_{k-1}\right) g\left(x_{k}-y_{k}\right) \\
& +w^{2} \sum_{s, t} \chi_{a}(s-t) F_{k+1}\left(X_{k}, s ; Y_{k-1}, t, s\right) g\left(t-y_{k}\right) \\
& +S^{(a)}\left(X_{k} ; Y_{k}\right)
\end{aligned}
$$

where

$$
\chi_{a}(x)= \begin{cases}R^{-d} \phi^{2}(x / R), & a=R \\ \delta(x), & a=n, \\ (2 d)^{-1} \delta(x-1), & a=d\end{cases}
$$

$\delta(x)$ is the Kronecker symbol, and for some $\eta_{0}>0$ independent of $a$ and $\eta=|\operatorname{Im} z| \geq$ $\eta_{0}$

$$
\left\|S^{(a)}\right\|_{k}^{(a)} \leq C k \eta^{-k} \begin{cases}a^{-1}, & a=R, n \\ a^{-1 / 2}, & a=d .\end{cases}
$$

Remark. We use here and below the common symbol $C$ for quantities, independent of $a, k, \eta$, but dependent, generally speaking, on the moments of $W(x, y)$, the function $\phi(x)$ and $\eta_{0}$.

Proof. We will need several simple auxiliary facts.

(i) Let $A, B$ and $A+B$ be selfadjoint operators and $B$ be bounded. Then, for $\operatorname{Im} z \neq 0$,

$$
\begin{aligned}
(A+B-z)^{-1} & =(A-z)^{-1}-(A+B-z)^{-1} B(A-z)^{-1}, \\
\left\|(A-z)^{-1}\right\| & \leq \eta^{-1}, \quad \eta=|\operatorname{Im} z| .
\end{aligned}
$$

Therefore, if $G(x, y) \equiv(A-z)^{-1}(x, y)$ is the Green function of a selfadjoint operator $A$ acting in $\ell^{2}\left(\mathbb{Z}^{d}\right)$, then

$$
\begin{aligned}
|G(x, y)| & \leq\left\|(A-z)^{-1}\right\| \leq \eta^{-1}, \\
\sum_{x}|G(x, y)|^{2} & =\left(G^{*} G\right)(x, x) \leq \eta^{-2}, \quad \sum_{y}|G(x, y)|^{2} \leq \eta^{-2} .
\end{aligned}
$$

(ii) Let $h$ be the selfadjoint Toeplitz operator in $\ell^{2}\left(\mathbb{Z}^{d}\right)$ defined by the matrix $h(x-y)$ with a function $h(x)$, that satisfies $(2.1 b)$, and $g(x-y)$ be the Green function of this operator. Then,

$$
\bar{g} \equiv \sum_{x}|g(x)| \leq 2 \eta^{-1} \leq 1 / \widehat{h}
$$

if

$$
\bar{h} \equiv \sum_{x}|h(x)|, \quad \eta \geq \eta_{0} \geq 2 \bar{h} .
$$

Indeed, the resolvent identity (3.11) with $A=0$ and $B=h$ yields

$$
g(x)=-z^{-1} \delta(x)+z^{-1} \sum_{y} g(x-y) h(y),
$$


where $\delta(x)$ is the Kronecker symbol. Thus, $\bar{g} \leq \eta^{-1}+\eta^{-1} \bar{g} \bar{h}$. This inequality implies (3.12).

(iii) Let $\xi$ be a Gaussian random variable with $\mathbb{E}\{\xi\}=0$ and $f(t), t \in \mathbb{R}$ be a bounded function. Then,

$$
\mathbb{E}\{\xi f(\xi)\}=\mathbb{E}\left\{\xi^{2}\right\} \mathbb{E}\left\{f^{\prime}(\xi)\right\} .
$$

This formula can be proved by integrating by parts.

(iv) If $G(x, y)$ is the Green function of a selfadjoint operator $H_{0}+V$ acting in $\ell^{2}\left(\mathbb{Z}^{d}\right)$, and the matrix $V(x, y)$ of $V$ is real-valued, then for $s \neq t$,

$$
\frac{\partial G(x, y)}{\partial V(s, t)}=-G(x, s) G(t, y)-G(x, t) G(s, y) \text {. }
$$

This formula follows easily from (3.8).

We start our derivation of (3.7) from the case of $a=R$.

Consider $F_{1}\left(x_{1} ; y_{1}\right) \equiv \mathbb{E}\left\{G\left(x_{1}, y_{1}\right)\right\}$, where $G(x, y)$ is the Green function of (2.1). By using the resolvent identity (3.8) with $A$ and $B$ equal to the nonrandom and random parts of (2.1) respectively, we have

$$
G(x, y)=g(x-y)-R^{-d / 2} \sum_{s, t} G(x, s) W(s, t) \phi((s-t) / R) g(t-y) .
$$

Notice that, rigorously speaking, (3.15) is not just the matrix form of (3.8). Indeed, the latter was written only for a bounded $B$, while in our case we have an unbounded operator because of the Gaussian nature of $W(x, y)$ in $(2.1)$. However, we will not justify this step here. This will be done later for arbitrary distributed $W$ 's, having a finite second moment (see Lemma 3.1 below).

By averaging (3.15) and using (2.1e), (3.13) and (3.14), we obtain

$$
\begin{aligned}
F_{1}\left(x_{1} ; y_{1}\right)= & g\left(x_{1}-y_{1}\right)+w^{2} R^{-d} \sum_{s, t} \phi^{2}((s-t) / R) \\
& \times \mathbb{E}\left\{G\left(x_{1}, t\right) G(s, s)+G\left(x_{1}, s\right) G(t, s)\right\} g\left(t-y_{1}\right) .
\end{aligned}
$$

In view of (3.4) this relation can be written in the form (3.5a) with

$$
S_{1}\left(x_{1} ; y_{1}\right) \equiv w^{2} R^{-d} \sum_{s, t} \phi^{2}((s-t) / R) \mathbb{E}\left\{G\left(x_{1}, s\right) G(t, s)\right\} g\left(t-y_{1}\right)
$$

But according to the Schwarz inequality and (3.11),

$$
\left|\sum_{s} G\left(x_{1}, s\right) G(t, s)\right| \leq\left[\sum_{s}\left|G\left(x_{1}, s\right)\right|^{2} \sum_{s}|G(t, s)|^{2}\right]^{1 / 2} \leq \eta^{-2} \text {. }
$$

This bound, (2.1c) and (3.12) yield for $\eta \geq \eta_{0}$ :

$$
\begin{aligned}
\left|S_{1}\left(x_{1} ; y_{1}\right)\right| & \leq w^{2} \phi_{0}^{2} R^{-d} \sum_{t}\left|g\left(t-y_{1}\right)\right|\left|\sum_{s} G\left(x_{1}, s\right) G(t, s)\right| \\
& \leq 2 w^{2} \phi_{0}^{2} \eta^{-3} R^{-d}
\end{aligned}
$$

We have arrived at (3.5a).

The two simple inequalities (3.18) and (3.19) are the essence of all subsequent derivations of (3.5)-(3.7). They show that terms which contain sums with Green functions, having noncoinciding arguments, vanish in the limits $a \rightarrow \infty$. 
We demonstrate now the same mathematical mechanism by deriving higher equations (3.5b). To this end, we replace again the last factor $G\left(x_{k}, y_{k}\right)$ in (3.4) by the resolvent identity (3.15) and use again (3.13) and (3.14). We obtain (3.5b) with

$$
S_{k}\left(X_{k} ; Y_{k}\right)=S^{(1)}\left(X_{k} ; Y_{k}\right)+\sum_{i=1}^{k-1} S_{i}^{(2)}\left(X_{k} ; Y_{k}\right)
$$

where

$$
\begin{aligned}
S^{(1)}\left(X_{k} ; Y_{k}\right)= & w^{2} R^{-d} \sum_{s, t} \phi^{2}((s-t) / R) \\
& \times \mathbb{E}\left\{G_{1} \ldots G_{k-1} G\left(x_{k}, s\right) G(t, s)\right\} g\left(t-y_{k}\right), \\
S_{i}^{(2)}\left(X_{k} ; Y_{k}\right)= & w^{2} R^{-d} \sum_{s, t} \phi^{2}((s-t) / R) \mathbb{E}\left\{G_{1} \ldots G_{i-1}\right. \\
& \times\left[G\left(x_{\imath}, s\right) G\left(t, y_{i}\right)+G\left(x_{\imath}, t\right) G\left(s, y_{i}\right)\right] \\
& \left.\times G_{i+1} \ldots G_{k-1} G\left(x_{k}, s\right)\right\} g\left(t-y_{k}\right),
\end{aligned}
$$

and $G_{i}=G\left(x_{i}, y_{i}\right)$.

Using (3.10), (3.11) and (3.12), we have:

$$
\begin{aligned}
\left|S_{k}^{(1)}\right| & \leq w^{2} \phi_{0}^{2} \eta^{-k+1} R^{-d} \sum_{t}\left|g\left(t-y_{k}\right)\right| \mathbb{E}\left\{\left|\sum_{s} G\left(x_{k}, s\right) G(t, s)\right|\right\} \\
& \leq 2 w^{2} \phi_{0}^{2} \eta^{-k-2} R^{-d} .
\end{aligned}
$$

The $i^{\text {th }}$ term in (3.22) can be estimated similarly by using the bound

$$
\eta^{-k+1} \mathbb{E}\left\{\left|G\left(x_{\imath}, s\right) G\left(x_{k}, s\right)\right|+\left|G\left(s, y_{i}\right) G\left(x_{k}, s\right)\right|\right\}
$$

for the expectation. The resulting bound for (3.20) is

$$
\left|S_{k}\right| \leq 4 w^{2} \phi_{0}^{2} k \eta^{-k-2} R^{-d}
$$

and satisfies (3.7).

The same arguments are valid also in the cases of $a=n, d$. We demonstrate them by deriving (3.5a) for $a=n$, $d$. For $a=n$ we have from the respective resolvent identity:

$$
\begin{aligned}
F_{1}\left(x_{1} ; y_{1}\right) & \equiv n^{-1} \sum_{\alpha=1}^{n} \mathbb{E}\left\{G\left(\alpha, x_{1} ; \alpha, y_{1}\right)\right. \\
& =g\left(x_{1}-y_{1}\right)-n^{-3 / 2} \sum_{\alpha, \beta=1}^{n} \sum_{s} \mathbb{E}\left\{G\left(\alpha, x_{1} ; \beta, s\right) W_{\beta \alpha}(s)\right\} g\left(s-y_{1}\right) .
\end{aligned}
$$

By applying the analogs of (3.13) and (3.14), we obtain (3.5a) with

$$
S_{1}\left(x_{1} ; y_{1}\right)=w^{2} n^{-2} \sum_{\alpha, \beta, s} \mathbb{E}\left\{G\left(\alpha, x_{1} ; \beta, s\right) G(\alpha, s ; \beta, s)\right\} g\left(t-y_{1}\right) .
$$

Now [cf. (3.18)]

$$
\left|\sum_{\beta} G(\alpha, x ; \beta, s) G(\alpha, s ; \beta, s)\right| \leq\left[\sum_{\beta}|G(\alpha, x ; \beta, s)|^{2} \sum_{\beta}|G(\alpha, s ; \beta, s)|^{2}\right]^{1 / 2} .
$$


Denote by $P_{x}$ the orthogonal projection projecting our space $\ell^{2} \otimes \mathbb{C}^{n}$ onto the subspace spanned by vectors $\psi(\alpha, t)=\psi_{\alpha} \delta(x-t)$, concentrated at $x$. Then the $n \times n$ matrix

$$
R(x, y)=\{G(\alpha, x ; \beta, y)\}_{\alpha, \beta=1}^{n}
$$

can be represented as follows:

$$
R(x, y)=P_{x}\left(H^{(n)}-z\right)^{-1} P_{y} .
$$

Thus, if $|R(x, y)|$ denotes the matrix norm of (3.27), we have from (3.10),

$$
|R(x, y)| \leq \eta^{-1}
$$

Besides, since the r.h.s. of (3.26) can be written as

$$
\left(\left[R(x, s) R^{*}(s, x)\right]_{\alpha \alpha}\left[R(s, s) R^{*}(s, s)\right]_{\alpha \alpha}\right)^{1 / 2},
$$

it admits the bound

$$
|R(x, s)||R(s, s)| \leq \eta^{-2} .
$$

As a result (cf. (3.19)), we obtain the inequality

$$
\left|S_{1}^{(n)}(x ; y)\right| \leq w^{2} \eta^{-2} n^{-1} \sum_{t}|g(t)| \leq 2 w^{2} \eta^{-3} n^{-1},
$$

coinciding with (3.7) for $a=n$.

The case of $a=d$ is more complicated. The reason is that due to the presence of the normalization factor $d^{-1 / 2}$ in $(2.2 \mathrm{~b})$, introduced to obtain the nontrivial limit (2.17) for the IDS of the translationally invariant part of (2.2), the quantity $\bar{h}$ in (3.12) has the order $d^{1 / 2}$ and $\eta_{0}$ cannot be chosen to be independent of $d$. Therefore we have at our disposal only the general and weaker bound

$$
\sum_{x}|g(x)|^{2} \leq \eta^{-2}
$$

that is a particular case of (3.11).

The analog of (3.21) is

$$
\begin{aligned}
& S^{(1)}\left(X_{k} ; Y_{k}\right) \\
& \quad=w^{2}(2 d)^{-1} \sum_{s, t} \delta(s-t-1) \mathbb{E}\left\{G_{1} \ldots G_{k-1} G\left(x_{k}, s\right) G(t, s)\right\} g\left(t-y_{k}\right) .
\end{aligned}
$$

While working with the case of $a=d$, we will often use the inequality

$$
\sum_{y}\left|\sum_{t} u(t) g(t-y)\right|^{2} \leq \eta^{-2} \sum_{t}|u(t)|^{2} .
$$

This inequality follows from the fact that its 1.h.s. is $\|g u\|^{2}$, where $g$ is the resolvent of the nonrandom Toeplitz part of (2.2), and therefore

$$
\|g u\|^{2} \leq\|g\|^{2}\|u\|^{2} \leq \eta^{-2}\|u\|^{2}
$$

where we have again used inequality (3.9). Setting

$$
u(t) \equiv w^{2}(2 d)^{-1} \sum_{s} \delta(s-t-1) \mathbb{E}\left\{G_{1} \ldots G_{k-1} G\left(x_{k}, s\right) G(t, s)\right\}
$$


we have, according to the Schwarz inequality for expectations,

$$
\begin{aligned}
u(t) \mid \leq & w^{2}(2 d)^{-1} \mathbb{E}^{1 / 2}\left\{\left|G_{1} \ldots G_{k-1}\right|^{2}\right\} \mathbb{E}^{1 / 2} \\
& \times\left\{\left|\sum_{s} \delta(s-t-1)\right| G\left(x_{k}, s\right) G(t, s)||\right\} .
\end{aligned}
$$

Now, applying the Schwarz inequality to the sum over $s$, we get

$$
\begin{aligned}
\left|\sum_{s} \delta(s-t-1)\right| G\left(x_{k}, s\right) G(t, s)||^{2} & \leq \sum_{s} \delta^{2}(s-t-1)\left|G\left(x_{k}, s\right)\right|^{2} \sum_{s}|G(t, s)|^{2} \\
& \leq \eta^{-2} \sum_{s} \delta^{2}(s-t-1)\left|G\left(x_{k}, s\right)\right|^{2}
\end{aligned}
$$

Combining (3.33), (3.34), (3.35) and (3.36), we obtain

$$
\begin{aligned}
\left(\sum_{y_{k}}\left|S^{(1)}\right|^{2}\right)^{1 / 2} & \leq w^{2}(2 d)^{-1} \eta^{-2} \mathbb{E}^{1 / 2}\left\{\left|G_{1} \ldots G_{k-1}\right|^{2}\right\} \mathbb{E}^{1 / 2}\left\{\sum_{s}\left|G\left(x_{1}, s\right)\right|^{2} \sum_{t} \phi^{2}(s-t)\right\} \\
& \leq w^{2}(2 d)^{-1 / 2} \eta^{-2} \mathbb{E}^{1 / 2}\left\{\left|G_{1} \ldots G_{k-1}\right|^{2}\right\}
\end{aligned}
$$

where we have also used (3.6) and (3.11).

Now, again taking into account (3.11), we obtain for the norm (3.1), $a=d$ :

$$
\left\|S^{(1)}\right\|_{k}^{(d)} \leq w^{2}(2 d)^{-1 / 2} \eta^{-k-2} .
$$

Similarly, to estimate the analog of (3.22) for $a=d$, we write for the sum over $s$ in the first term, containing $G\left(x_{i}, s\right) G\left(t, y_{i}\right)$ :

$$
\begin{aligned}
& \left|\sum_{s} \phi(s-t) \mathbb{E}\left\{G_{1} \ldots G_{i-1} G\left(x_{\imath}, s\right) G\left(t, y_{\imath}\right) G_{i+1} \ldots G_{k-1} G\left(x_{k}, s\right)\right\}\right| \\
& \leq\left(\sum_{s} \delta^{2}(s-t-1) \mathbb{E}\left\{\left|G_{1} \ldots G_{k-1}\right|^{2}\right\} \mathbb{E}\left\{\sum_{s}\left|G\left(x_{k}, s\right)\right|^{2}\right\}\right)^{1 / 2}
\end{aligned}
$$

Since the second factor in the r.h.s. is bounded from above by $\eta^{-2}$ we see after applying (3.33) that the $\ell^{2}$ - norm with respect to $y_{k}$ of the corresponding term does not exceed

$w^{2}(2 d)^{-1} \eta^{-2}\left(\sum_{s, t} \delta^{2}(s-t-1) \mathbb{E}\left\{\left|G_{1} \ldots G_{\imath-1} G\left(x_{i}, s\right) G\left(t, y_{\imath}\right) G_{i+1} \ldots G_{k-1}\right|^{2}\right\}\right)^{1 / 2}$.

Now, calculating the norm (3.1) for $a=d$ of this expression with respect to the variables $y_{1}, x_{1}, \ldots, y_{k-1}, x_{k-1}$ by using (3.10) and (3.11), we find that this norm is bounded by the r.h.s. of (3.38). The same bound is valid for the second term containing $G\left(x_{i}, t\right) G\left(s, y_{\imath}\right)$. Combining all these bounds, we get (3.7) for $a=d$. Proposition 3.1 is proved.

Proposition 3.2. Let $H_{a}, a=R, n, d$ be defined in (2.1)-(2.3), where the arbitrarily distributed i.i.d. W's satisfy $(2.1 e, f)$ and $(2.3 c, d)$ and, in addition,

$$
\mathbb{E}\left\{|W(x, y)|^{3}\right\}<\infty, \quad \mathbb{E}\left\{\left|W_{\alpha, \beta}(x)\right|^{3}\right\}<\infty .
$$


Assume also that $h(x) \equiv 0$ in (2.2a). Then, the moments (3.4) satisfy (3.5) and (33.6), where

$$
\sup _{X_{k}, Y_{k}}\left|S_{k}\left(X_{k} ; Y_{k}\right)\right| \leq C k a^{-1 / 2} \eta^{-k}
$$

with $\eta \geq \eta_{0}$, some $\eta_{0}$ and $C$ being independent of $k, \eta$ and $a$.

We prove first our basic relation.

Lemma 3.1. The Relation

$$
\begin{aligned}
& F_{k}\left(X_{k} ; Y_{k}\right) \\
&= F_{k-1}\left(X_{k-1} ; Y_{k-1}\right) g\left(x_{k}-y_{k}\right) \\
&-\sum_{s, t} \chi_{a}(s-t) \mathbb{E}\left\{G\left(x_{1}, y_{1}\right) \ldots G\left(x_{k-1}, y_{k-1}\right) G\left(x_{k}, s\right) W(s, t)\right\} g\left(t-y_{k}\right)
\end{aligned}
$$

is valid for the moments (3.4) of the quantities (3.2), with the convention that $F_{0} \equiv 1$ and $\chi_{a}$ given by (3.6). For $a=n$ the factor $G\left(x_{k}, s\right) W(s, t)$ in (3.41) should be replaced by

$$
\delta(s-t) n^{-2} \sum_{\alpha, \beta} G\left(\alpha, x_{k} ; \beta, t\right) W_{\beta \alpha}(t) .
$$

Proof. We give a detailed proof only for $a=R$, because other cases are quite similar. Note first of all that all terms of this relation are well defined under the conditions (2.1e,f). Indeed, according to [25], (2.1) is an essentially selfadjoint operator with probability 1 on a linear manifold, consisting of $\ell^{2}$-vectors with finitely many nonzero components. Thus, the Green functions $G_{a}\left(x_{i}, y_{i}\right)$ are bounded operators for $\operatorname{Im} z \neq 0$ and satisfy (3.10). Therefore, all moments $F_{k}\left(X_{k} ; Y_{k}\right), k \geq 1$, exist and

$$
\left|F_{k}\left(X_{k} ; Y_{k}\right)\right| \leq \eta^{-k}, \quad \eta=|\operatorname{Im} z| .
$$

Besides, according to the Schwarz inequality,

$$
\begin{aligned}
& \left|R^{-d / 2} \sum \phi((s-t) / R) \mathbb{E}\left\{G_{1} \ldots G_{k-1} G\left(x_{k}, s\right) W(s, t)\right\} g\left(t-y_{k}\right)\right| \\
& \quad \leq w^{2} R^{-d / 2} \eta^{-k /+1} \sum_{s, t} \phi((s-t) / R) \mathbb{E}^{1 / 2}\left\{\left|G\left(x_{k}, s\right)\right|^{2}\right\}\left|g\left(t-y_{k}\right)\right| \\
& \quad \leq w^{2} \phi_{1} R^{d / 2} \eta^{-k-1}
\end{aligned}
$$

where we used (2.1c), (3.10), (3.11) and the inequality

$$
\left|R^{-d} \sum_{s, t} \phi((s-t) / R) a(s) b(t)\right| \leq \phi_{1}\left[\sum_{s}|a(s)|^{2} \sum_{t}|b(t)|^{2}\right]^{1 / 2}
$$

with

$$
\phi_{1} \equiv R^{-d} \sum_{x} \phi(x / R)<\infty
$$

Thus, all terms of (3.41) are well defined if $W(s, t), s, t \in \mathbb{Z}^{d}$ have a finite second moment and $a=R$ is finite. The same conclusion can be made for $a=n, d$. 
Therefore we are to prove coincidence of the 1.h.s. and r.h.s. parts of this relation for $\operatorname{Im} z \neq 0$. To do so, we will show that (3.41) is the limit of a similar relation, written for truncated $W^{\prime}$ 's, defined as

$$
W_{T}(s, t)= \begin{cases}W(s, t), & |W(s, t)| \leq T \\ 0, & |W(s, t)|>T\end{cases}
$$

for some $T<\infty$.

Since for bounded $W$ 's the random parts of (2.1)-(2.3) are bounded operators, the resolvent identity (3.15) and its obvious analogs for $a=n, d$ are also valid.

If $H_{a}^{T}$ are the corresponding operators (2.1)-(2.3), then with probability 1 they converge strongly to $H$ as $T \rightarrow \infty$ for all vectors with finitely many nonzero components. Since $H_{a}$ are essentially selfadjoint on this linear manifold (see [25]), the resolvent of $H_{a}^{T}$ converge strongly to the resolvents of $H_{a}$ as $T \rightarrow \infty$ [13]. Thus, with probability 1 and for fixed $x, y \in \mathbb{Z}^{d}$,

$$
\lim _{T \rightarrow \infty} G_{a}^{T}(x, y)=G_{a}(x, y)
$$

This relation and the uniformity in $T$ of the bound (3.10) imply that

$$
\lim _{T \rightarrow \infty} F^{T}\left(X_{k} ; Y_{k}\right)=F\left(X_{k} ; Y_{k}\right)
$$

for all $k \geq 1$ and fixed $X_{k} Y_{k}$. Thus, we can perform the limiting transition $T \rightarrow \infty$ in the l.h.s. of (3.41) and in the first term of the r.h.s.. Besides, we have pointwise convergence of the expectation in the second term of the r.h.s.. Since the estimate (3.44) implies convergence of the sum over $s$ and $t$ uniform in $T \rightarrow \infty$ for every fixed $R<\infty$, we can also perform a limiting transition in the second term of the r.h.s. of (3.41).

The lemma is proved.

Proof of Proposition 3.2. According to Lemma 3.1, we have relation (3.41) for the finite second moment of $W$ 's. Let us write now the resolvent identity (3.8) for $A+B=H_{R}$ and $A=\left.H_{R}\right|_{W(s, t)=0} \equiv \hat{H}_{R}$,

$$
\begin{aligned}
G(x, y)= & \hat{G}(x, y)-R^{-d / 2}[\hat{G}(x, s) G(t, y)+\hat{G}(x, t) G(s, y)] \\
& \times \hat{W}(s, t) \phi(s-t) \\
\hat{W}(s, t)= & \alpha(s, t) W(s, t), \quad \alpha(s, t)= \begin{cases}1 / 2, & s=t \\
1, & s \neq t\end{cases}
\end{aligned}
$$

where $\hat{G}=\left.G\right|_{W(s, t)=0}$. Replacing by the r.h.s. of (3.50) with $x=x_{k}, y=s$ the factor $G\left(x_{k}, s\right)$ in the second term of the r.h.s. of (3.41), we obtain for this term

$$
\begin{aligned}
R^{-d / 2} \sum_{s, t} \phi((s-t) / R) \mathbb{E}\left\{G_{1} \ldots G_{k-1} G\left(x_{k}, s\right) \hat{W}(s, t)\right\} g\left(t-y_{k}\right) \\
=-A_{k}\left(X_{k} ; Y_{k}\right)+B_{k}\left(X_{k} ; Y_{k}\right)-C_{k}\left(X_{k} ; Y_{k}\right) .
\end{aligned}
$$


Here

$$
\begin{aligned}
A_{k}= & R^{-d} \sum_{s, t} \phi^{2}((s-t) / R) \\
& \times \mathbb{E}\left\{G_{1} \ldots G_{k-1} \hat{G}\left(x_{k}, t\right) G(s, s) \hat{W}^{2}(s, t)\right\} g\left(t-y_{k}\right) \\
B_{k}= & R^{-d / 2} \sum_{s, t} \phi^{2}((s-t) / R) \mathbb{E}\left\{G_{1} \ldots G_{k-1} \hat{G}\left(x_{k}, s\right) \hat{W}(s, t)\right\} g\left(t-y_{k}\right) \\
C_{k}= & R^{-d} \sum_{s, t} \phi^{2}((s-t) / R) \\
& \times \mathbb{E}\left\{G_{1} \ldots G_{k-1} \hat{G}\left(x_{k}, s\right) G(t, s) \hat{W}^{2}(s, t)\right\} g\left(t-y_{k}\right)
\end{aligned}
$$

and $G \equiv G\left(x_{i}, y_{i}\right), i=1, \ldots, k-1$.

We are to show now that $B_{k}$ and $C_{k}$ are of the order $O\left(R^{-d / 2}\right)$ and $A_{k}$ with the same accuracy can be written in the form of the second term in the r.h.s. of (3.5b). Since $\mathbb{E}\{W(s, t)\}=0$ and $W(s, t)$ are independent except the symmetry condition (2.1d), the expectation in (3.53) with all $G$ 's replaced by $\hat{G}$ 's is zero. Thus, by using the elementary inequality

$$
\prod_{i=1}^{k-1} a_{i}-\prod_{i=1}^{k-1} b_{i}=\sum_{i=1}^{k-1} a_{1} \ldots a_{i-1}\left(a_{i}-b_{i}\right) b_{i+1} \ldots b_{k-1},
$$

we rewrite (3.53) in the form

$$
\begin{aligned}
B_{k}= & R^{-d / 2} \sum_{i=1}^{k-1} \sum_{s, t} \phi((s-t) / R) \\
& \times \mathbb{E}\left\{G_{1} \ldots G_{i-1} \Delta \hat{G}_{i} \hat{G}_{i+1} \ldots \hat{G}_{k} \hat{W}(s, t)\right\} g\left(t-y_{k}\right),
\end{aligned}
$$

where

$$
\Delta G_{\imath} \equiv G_{i}-\hat{G}_{\imath}
$$

According to (3.50) and (3.10),

$$
\left|\Delta G_{i}\right| \leq 2 R^{-d / 2} \eta^{-2}|W(s, t)| \phi((s-t) / R) .
$$

This inequality, (3.10) and (2.1e,f) yield for (3.56):

$$
\begin{aligned}
\left|B_{k}\right| & \leq 2(k-1) R^{-d} \eta^{-k} \sum_{s, t} \phi^{2}((s-t) / R) \times \mathbb{E}\left\{\left|\hat{G}\left(x_{k}, s\right)\right| W^{2}(s, t)\right\} g\left(t-y_{k}\right) \\
& =2 w^{2}(k-1) R^{-d} \eta^{-k} \sum_{s, t} \phi^{2}((s-t) / R) \mathbb{E}\left\{\left|\hat{G}\left(x_{k}, s\right)\right|\right\}\left|g\left(t-y_{k}\right)\right| .
\end{aligned}
$$

Since $\hat{G}(x, y)$ depends on $s$ and $t$, we cannot use directly inequality (3.11). Thus, we apply once more (3.58) and obtain:

$$
\mathbb{E}\left\{\left|\hat{G}\left(x_{k}, s\right)\right|\right\} \leq \mathbb{E}^{1 / 2}\left\{\left|G\left(x_{k}, s\right)\right|^{2}\right\}+2 R^{-d / 2} \eta^{-2} \mathbb{E}\{|W(s, t)|\} \phi((s-t) / R) .
$$


By using this inequality in (3.59) we obtain for $\eta>\eta_{0}$ :

$$
\begin{aligned}
\left|B_{k}\right| \leq & 2 w^{2}(k-1) R^{-d} \eta^{-k} \sum_{t}\left|g\left(t-y_{k}\right)\right| \\
& \times\left[\left(\sum_{s} \phi^{4}((s-t) / R) \sum_{s} \mathbb{E}\left\{\left|G\left(x_{k}, s\right)\right|^{2}\right\}\right)^{1 / 2}\right. \\
& \left.+2 w R^{-d / 2} \eta^{-2} \sum_{s} \phi^{3}((s-t) / R)\right] \leq C(k-1) R^{-d / 2} \eta^{-k-2}
\end{aligned}
$$

where we have used (3.12) and (2.1c).

To find a bound for (3.54), we use (3.10) for all $G$ 's and the independence of $\hat{G}$ of $W(s, t)$ :

$$
\left|C_{k}\right| \leq w^{2} R^{-d} \eta^{-k} \sum_{s, t} \phi^{2}((s-t) / R) \mathbb{E}\left\{\left|\hat{G}\left(x_{k}, s\right)\right|\right\}\left|g\left(t-y_{k}\right)\right|
$$

The sum in this inequality coincides with that in (3.59). Thus, in view of (3.60) we have for $\eta>\eta_{0}$,

$$
\left|C_{k}\right| \leq C R^{-d / 2} \eta^{-k-2}
$$

Combining (3.60) and (3.61), we obtain that

$$
\left|B_{k}\right|+\left|C_{k}\right| \leq C k R^{-d / 2} \eta^{-k-2}
$$

where $C$ is independent of $k, \eta$ and $R$.

Consider now (3.52). To prove that this expression coincides with the second term of (3.5b) up to small terms, we use (3.50) to replace all $G$ 's by $\hat{G}$ 's, single out $\mathbb{E}\left\{W^{2}(s, t)\right\}=w^{2}$ and return back to $G$ 's using the same formula (3.50). To do so, we represent (3.52) as

$$
A_{k}=A_{k}^{\prime}+A_{k}^{\prime \prime}+\sum_{i=1}^{k-1} A_{i, k}
$$

where

$$
\begin{aligned}
A^{\prime}= & w^{2} R^{-d} \sum_{s, t} \phi^{2}((s-t) / R) \mathbb{E}\left\{\hat{G}_{1} \ldots \hat{G}_{k-1} \hat{G}\left(x_{k}, t\right) \hat{G}(s, s)\right\} g\left(t-y_{k}\right), \\
A^{\prime \prime}= & R^{-d} \sum_{s, t} \phi^{2}((s-t) / R) \\
& \times \mathbb{E}\left\{G_{1} \ldots G_{k-1} \hat{G}\left(x_{k}, s\right) \Delta G(s, s) W^{2}(s, t)\right\} g\left(t-y_{k}\right) \\
A_{\imath, k}= & R^{-d} \sum_{s, t} \phi^{2}((s-t) / R) \\
& \times \mathbb{E}\left\{G_{1} \ldots G_{i-1} \Delta G_{\imath} \hat{G}_{i+1} \ldots \hat{G}_{k-1} \hat{G}\left(x_{k}, s\right) \hat{G}(s, s) \hat{W}^{2}(s, t)\right\} g\left(t-y_{k}\right),
\end{aligned}
$$

and $\Delta G(x, y)$ is given by (3.57) and (3.50).

By using (3.5), (3.10), (3.12), (3.58) and (2.1c) we obtain

$$
\begin{aligned}
\left|A_{k}^{\prime \prime}\right| & \leq 2 w_{3} R^{-3 d / 2} \eta^{-k-2} \sum_{s, t} \phi^{3}((s-t) / R)\left|g\left(t-y_{k}\right)\right| \\
& \leq 4 w_{3} \phi_{0} \phi^{2} R^{-d / 2} \eta^{-k-3}
\end{aligned}
$$


where, according to (2.1c),

$$
\phi^{2} \equiv R^{-d} \sum_{x} \phi^{2}(x / R) \underset{R \rightarrow \infty}{\longrightarrow} 1
$$

The same bound is valid for (3.66). Thus, for $\eta \operatorname{Re} \eta_{0}$, where $\eta_{0}$ is specified in (3.12b), we have

$$
\left\|A^{\prime \prime}+\sum_{i=1}^{k-1} A_{\imath, k}\right\|_{k}^{(R)} \leq 4 w_{3} \phi_{0} \phi^{2} k \eta^{-k-3} \leq C k \eta^{-k} R^{-d / 2},
$$

where, as in (3.62), $C$ is independent on $k, \eta$, and $R$. The "inverse" procedure of replacing $\hat{G}$ 's by $G$ 's in (3.64) is quite similar to the "direct" procedure of replacing $G^{\prime} s$ by $\hat{G}$ 's that we carried out above. The only difference is that the latter requires finiteness of the first moment of $W$ 's but not the third one as above. Indeed, the replacement of $\hat{G}_{1}, \ldots, \hat{G}_{k-1}$ and $\hat{G}(s, s)$ in (3.64) by the respective $G$ 's is literally the same. Consider therefore the replacement of $\hat{G}\left(x_{k}, t\right)$ by $G\left(x_{k}, t\right)$ in (3.64). By using (3.10), (3.58) and (2.1e), we find for the respective remainder term,

$$
\begin{aligned}
& 2 w^{2} R^{-3 d / 2} \eta^{-k-2} \sum_{s, t} \phi^{3}((s-t) / R) \mathbb{E}\{|W(s, t)|\}\left|g\left(t-y_{k}\right)\right| \\
& \quad \leq 4 w^{3} \phi_{0} \phi^{2} R^{-d / 2} \eta^{-k-3}
\end{aligned}
$$

that is again the bound of the form of (3.61).

Combining all these bounds, we obtain (3.7). Thus, we have proved relations (3.5)-(3.7) for $a=R$.

Note, that at the same time we have obtained the proof for the case of $a=d$, because, according to our hypothesis, in this case $h(x)=0, g(x-y)=-z^{-1} \delta(x-y)$, and this function obviously satisfies (3.12) for any $\eta_{0}>0$. That is why we have assumed that $h(x)=0$ in this case. The proof of Proposition 3.2 for $a=d$ and $h(x) \neq 0$ is rather cumbersome and will be published elsewhere.

Derivation of relations (3.5)-(3.7) for $a=n$ is quite similar.

\section{Asymptotic Solution of the Moment Equations}

In the previous section we proved that the moments (3.4) of the Green functions of the operators (2.1)-(2.3) satisfy the infinite system of relations (3.5). In this section we treat this system as a linear equation in some Banach space and prove that for $a \rightarrow \infty$ this equation admits a rather simple solution. As a result, we obtain (2.15).

Theorem 4.1. Let $G^{(a)}(x, y ; z)$ be defined in (3.2), the respective operators $H^{(a)}$ satisfy the conditions of Proposition 3.1 in the case of Gaussian W's and the conditions of Proposition 3.2 in the general case and

$$
\eta_{1}=\max \{4 w, 2 \bar{h}\}, \quad \xi= \begin{cases}\frac{3}{2} w, & a=R, n \\ w, & a=d\end{cases}
$$

If a is large enough, then

$$
\sup _{|\operatorname{Im} z| \geq \eta_{1}}\left\|\mathbb{E}\left\{\prod_{i=1}^{k} G_{a}\left(x_{i}, y_{i} ; z\right)\right\}-\prod_{i=1}^{k} \mathscr{G}_{a}\left(x_{i}-y_{i} ; z\right)\right\|_{k}^{(a)} \leq C \xi^{-k} a^{-1 / 2} .
$$


Here $k \geq 1, C$ is independent of $k$ and $a$,

$$
\mathscr{G}_{a}(x ; z)=\int_{\mathbb{T}^{d}}\left[\tilde{h}(k)-z-w^{2} \chi_{a} r_{a}(z)\right]^{-1} \exp (2 \pi i k x) d k,
$$

where $\tilde{h}(k)$ is given by (2.13),

$$
\chi_{a} \equiv \sum_{x} \chi_{a}(x)= \begin{cases}\phi^{2}, & a=R \\ 1, & a=n, d\end{cases}
$$

$\phi^{2}$ is given by (3.46) and $r_{a}(z)$ is a unique solution of the equation

$$
r_{a}(z)=\int\left[\mu-z-w^{2} \chi_{a} r_{a}(z)\right]^{-1} N_{0}(d \mu),
$$

in the class of functions analytic in $z$ for nonreal $z$ and such that

$$
\begin{gathered}
\operatorname{Im} r(z) \cdot \operatorname{Im} z \geq 0, \quad \operatorname{Im} z \neq 0 \\
\sup _{\eta>0} \eta|r(i \eta)|=1
\end{gathered}
$$

and $N_{0}(d \mu)$ is specified by (2.12) for $a=R, n$ and (2.17) for $a=d$.

Proof. Let us consider the Banach space $\mathscr{B}_{a}, a=R, n, d$ whose elements are sequences

$$
f=\left\{f_{k}\left(X_{k} ; Y_{k} ; z\right)\right\}_{k \geq 1}
$$

$X_{k}=\left(x_{1}, \ldots, x_{k}\right), Y_{k}=\left(y_{1}, \ldots, y_{k}\right), x_{\imath}, y_{\imath} \in \mathbb{Z}^{d}$, and each component $f_{k}$ for fixed $k, X_{k}, Y_{k}$ is an analytic function of $z$ for nonreal $z$. The norm in $\mathscr{B}_{a}$ is defined as follows:

$$
\|f\|^{(a)}=\sup _{|\operatorname{Im} z| \geq \eta_{1}} \sup _{k \geq 1} \xi^{k}\left\|f_{k}\right\|_{k}^{(a)}
$$

According to (3.10) and (3.11), the moments (3.4) satisfy the inequalities

$$
\left\|F_{k}\right\|_{k} \leq \eta^{-k}, \quad k \geq 1 \text {. }
$$

Since $\xi<\eta_{1}$, the sequence $F \equiv\left\{F_{k}\right\}_{k \geq 1}$ of the moments (3.4) belongs to $\mathscr{B}_{a}$. Now, according to (3.19) and (3.24), the sequence $S=\left\{S_{k}\right\}_{k \geq 1}$ of the remainders in (3.5b) for $\eta \geq \eta_{0}$ satisfies the inequality

$$
\|S\|^{(a)} \leq C a^{-1 / 2} \sup _{k \geq 1} k \xi^{-k} \eta_{0}^{-k}
$$

Since our bounds in Proposition 3.1 are monotone in $\eta_{0}$ (under the condition (3.12a)), we can assume without loss of generality that $\eta_{0}=\max \left(\eta_{1}, 2 \bar{h}\right)$, where $\bar{h}$ is given by (3.12). Then, $\xi \cdot \eta_{0}^{-1}<1$, and (4.11) implies that

$$
\|S\|^{(a)} \leq C_{1} a^{-1 / 2}
$$

where $C_{1}$ is independent of $a$. 
Consider now the linear operator $\mathscr{A}$, defined by the first and second terms in the r.h.s. of (3.5a) and (3.5b):

$$
(\mathscr{f} f)_{k}\left(X_{k}, Y_{k}\right)= \begin{cases}\sum_{s, t} \chi_{a}(s-t) f_{2}\left(x_{1}, s ; t, s\right) g\left(t-y_{k}\right), & k=1 \\ f_{k-1}\left(X_{k-1} ; Y_{k-1}\right) g\left(x_{k}-y_{k}\right) & \\ +w^{2} \sum_{s, t} \chi_{a}(s-t) f_{k+1}\left(X_{k}, s ; Y_{k-1}, t, s\right) g\left(t-y_{k}\right), & k \geq 2 .\end{cases}
$$

We prove in Lemma 4.1 below that

$$
\|A\| \leq 3 / 4
$$

if $a$ is large enough.

Therefore we can regard relations (3.7) as a linear relation in $\mathscr{B}_{a}$ :

$$
F=\mathscr{A} F+U+S
$$

where $U=\left\{g\left(x_{1}, y_{1}\right), 0, \ldots\right\}$ belongs obviously to $\mathscr{B}_{a}$. Since, according to (4.11), $S$ is small for $a \rightarrow \infty$, it is natural to consider the following linear equation in $\mathscr{S}_{a}$ :

$$
J=A J+U \text {. }
$$

The bound (4.14) implies that this equation has a unique solution in $\mathscr{B}_{a}$. The ansatz

$$
J_{k}\left(X_{k} ; Y_{k}\right)=\prod_{i=1}^{k} \mathscr{G}\left(x_{i}-y_{i}\right)
$$

reduces the infinite system (4.16) to the single equation

$$
\mathscr{G}(x-y)=\gamma(x-y)+w^{2} \sum_{s, t} \chi_{a}(s-t) \mathscr{G}(0) \mathscr{G}(x-t) g(t-y) .
$$

The formal solution of this equation is

$$
\mathscr{G}(x-y)=g\left(x-y ; z+w^{2} \chi_{a} \mathscr{G}(0 ; z)\right),
$$

where $\chi_{a}$ is specified by (4.4) and the dependence on the complex energy $z$ is indicated explicitly. Equation (4.19) is valid under the compatibility condition

$$
\mathscr{G}(0 ; z)=g\left(0 ; z+w^{2} \chi_{a} \mathscr{G}(0 ; z)\right)
$$

which, in view of (2.12) and (3.3), can be rewritten in the form (4.5).

In Lemma 4.3 below we prove that Eq.(4.5) has a unique solution in the class of functions that are analytic for $\operatorname{Im} z \neq 0$ and satisfy (4.6) and (4.7). Therefore formulas (4.17)-(4.20) give the unique solution of the infinite system (4.16) in our spaces $\mathscr{B}_{a}$. Subtracting (4.16) from (4.15) and iterating the resulting relation, we find:

$$
F-J=(I-\mathscr{C})^{-1} S \text {. }
$$

Thus, in view of (4.12) and (4.14),

$$
\|F-J\|^{(a)} \leq 4 C_{1} a^{-1 / 2} .
$$

According to (4.9) and (4.17), the, $k^{\text {th }}$ component of (4.21) is (4.2). The theorem is proved. 
Corollary 4.1. For every fixed $k \geq 1, X_{k}=\left(x_{1} \ldots, x_{k}\right), Y_{k}=\left(y_{1}, \ldots, y_{k}\right)$ and uniformly in $z$ belonging to a fixed compact set such that $\operatorname{Im} z \neq 0$, we have

$$
\lim _{a \rightarrow \infty}\left(F_{k}\left(X_{k} ; Y_{k}\right)-\prod_{i=1}^{k} \mathscr{G}\left(x_{i}-y_{i}\right)\right)=0 .
$$

Proof. Theorem 4.1 and Definition (4.9) of the norm $\|\cdot\|^{(a)}$ yield the assertion of the Corollary for $|\operatorname{Im} z| \geq \eta_{1}$. Since, according to Definition (3.40) of the moments $F_{k}$, (4.17), (4.19) and Lemma 4.3 below, the difference $F_{k}-J_{k}$ is a bounded analytic function for $\operatorname{Im} z \neq 0$, then standard arguments of complex analysis complete the proof of the corollary.

Lemma 4.1. If. th is the linear operator in $\mathscr{B}_{a}$ defined by (4.13), then for sufficiently large $a$,

$$
\|\mathscr{b}\|^{(a)} \leq 3 / 4
$$

Proof. We start from the case of $a=R$. Here, according to (4.9), if $\|f\|=1$, then $\left|f_{k}\left(X_{k} ; Y_{k}\right)\right| \leq \xi^{-k}$ for $\eta \geq \eta_{1}$, where $\eta_{1}$ is given by (4.1). Thus, we have from (4.13), (3.68) and (3.12)

$$
\begin{aligned}
\left|(\mathscr{b} f)_{k}\left(X_{k} ; Y_{k}\right)\right| & \leq \eta^{-1} \xi^{-k+1}+\xi^{-k-1} w^{2} R^{-d} \sum_{s, t} \phi^{2}((s-t) / R)\left|g\left(t-y_{k}\right)\right| \\
& \leq \eta^{-1} \xi^{-k}\left(\xi+2 \phi^{2} w^{2} \xi^{-1}\right) .
\end{aligned}
$$

This implies that $\|\mathscr{A}\|^{(R)} \leq \eta_{1}^{-1}\left(\xi+2 \phi^{2} w^{2} \xi^{-1}\right)$. Selecting $\xi=\left(2 \phi^{2} w^{2} \xi^{-1}\right)^{1 / 2}$, we minimize this bound for the norm: $\|\mathscr{G}\|^{(R)} \leq \eta_{1}^{-1}\left(2^{3} \phi^{2} w^{2}\right)^{1 / 2}$. Since $\phi^{2} \rightarrow 1$ as $R \rightarrow \infty$, then $\|. \&\|^{(R)} \leq 3 \eta_{1}^{-1} w \leq 3 / 4$, if $R$ is large enough.

The case $a=n$ is quite similar.

Consider $a=d$. By using again inequality (3.33) to calculate the $\ell^{2}$-norm with respect to $y_{k}$ of the integral term in (3.1), we obtain

$$
\begin{aligned}
& \left(\sum_{y_{k}}\left|(\mathscr{C} f)_{k}\right|^{2}\right)^{1 / 2} \leq \eta^{-1}\left|f_{k-1}\left(X_{k-1} ; Y_{k-1}\right)\right| \\
& \quad+w^{2}(2 d \eta)^{-1}\left(\sum_{t}\left|\sum_{s} \delta(s-t-1) f_{k+1}\left(X_{k}, s ; Y_{k-1}, t, s\right)\right|^{2}\right)^{1 / 2} .
\end{aligned}
$$

But since

$$
\begin{aligned}
& \left|f_{k+1}\left(X_{k}, s ; Y_{k-1}, t, s\right)\right| \leq \hat{f}\left(X_{k} ; Y_{k-1}, t\right) \\
& \quad \equiv\left(\sup _{x_{k+1}} \sum_{y_{k+1}}\left|f_{k+1}\left(X_{k}, x_{k+1} ; Y_{k-1}, t, y_{k+1}\right)\right|^{2}\right)^{1 / 2}
\end{aligned}
$$

and this bound is independent of $s$, then we obtain

$$
\left(\sum_{y_{k}}\left|(\mathscr{b} f)_{k}\right|^{2}\right)^{1 / 2} \leq \eta^{-1}\left(\left|f_{k-1}\right|+w^{2}\left[\sum_{t} \hat{f}^{2}\left(X_{k} ; Y_{k-1}, t\right)\right]^{1 / 2}\right)
$$


This inequality implies that

$$
\left\|\mathscr{f} f_{k}\right\|_{k}^{(d)} \leq \eta_{1}^{-1}\left(\left\|f_{k-1}\right\|_{k-1}^{(d)}+w^{2}\left\|f_{k+1}\right\|_{k+1}^{(d)}\right) .
$$

If $\|f\|^{(d)}=1$, then, according to (4.9), $\left\|f_{k}\right\|_{k}^{(d)} \leq \xi^{-k}$ and as a result, $\|\mathscr{C}\|^{(d)} \leq$ $\eta_{1}^{-1}\left(\xi+w^{2} \xi^{-1}\right)$. This bound is minimal for $\xi=w$, when $\|. \mathscr{A}\|^{(d)} \leq 2 w \eta_{1}^{-1}$. Now, since $\eta_{1}=4 w$, we obtain the inequality $\|\mathscr{f}\|^{(d)} \leq 3 / 4$. The lemma is proved.

Lemma 4.2. The Equation

$$
f(z)=\int\left(t-z-w^{2} f(z)\right)^{-1} \mu_{0}(d t)
$$

with a nonnegative measure $\mu_{0}(d t)$, such that

$$
\mu_{0}(\mathbb{R})=1
$$

and $w^{2} \geq 0$, is uniquely solvable in the class of functions that can be represented in the form (2.9) with a nonnegative measure $\mu(d t)$ such that

$$
\mu(\mathbb{R})=1 .
$$

This solution for $\operatorname{Im} z \neq 0$ is continuous in $w^{2}$ and in $\mu_{0}(d t)$ (in the latter case we mean weak convergence of measures).

Proof. Consider the set of functions, having the form (2.9) with

$$
\mu(\mathbb{R})=1
$$

as a metric space $\mathscr{l} b$ with the distance

$$
\varrho\left(f_{1}, f_{2}\right)=\sup _{\eta \geq \eta_{1}} \eta\left|f_{1}(i \eta)-f_{2}(i \eta)\right|
$$

where

$$
\eta_{1}>2 w
$$

Since convergence in this metric implies convergence on any compact of the halfplane $\operatorname{Im} z \geq \eta_{1}$, then by using the Helly theorems and the uniqueness theorem for analytic functions it is easy to prove that $\mathscr{M}$ is complete. Define the operation

$$
(K f)(z)=\int\left(t-z-w^{2} f(z)\right)^{-1} \mu_{0}(d t) .
$$

According to [2], the class of functions specified by (2.9) and (4.26) coincides with the class of functions analytic for $\operatorname{Im} z \neq 0$ and satisfying (2.10). This allows us to check that $K$ maps $\mathscr{C} b$ into itself. Besides,

$$
\begin{aligned}
\varrho\left(K f_{1}, K f_{2}\right) & \leq w^{2} \sup _{\eta \geq \eta_{1}} \eta\left|\left(f_{1}(i \eta)-f_{2}(i \eta)\right) \int \frac{\mu_{0}(d t)}{\left(t-i \eta-w^{2} f_{1}(i \eta)\right)\left(t-i \eta-w^{2} f_{2}(i \eta)\right)}\right| \\
& \leq w^{2} \eta_{1}^{-2} \varrho\left(f_{1}, f_{2}\right) .
\end{aligned}
$$

Thus, under the condition (4.27), $K$ is a contraction. This proves existence and uniqueness of the solution of (4.23) of the form (2.9).

Now, it is easy to check that

$$
\varrho(0, f)=\sup _{\eta \geq \eta_{1}} \eta|f(i \eta)|=\mu(\mathbb{R}) .
$$


By using this relation in (4.23) we obtain that

$$
\mu(\mathbb{R})=\mu_{0}(\mathbb{R})=1 .
$$

At last, since under the condition (4.27) Eq. (4.23) is uniquely solvable uniformly with respect to small variations of $w$ and $\mu_{0}(d t)$, then we arrive at the last assertion of the lemma.

Remarks.

(1) It is easy to see that in fact we have proved the assertion similar to Theorem 4.1 and Corollary 4.1 for the sequence of moments more general than (3.4),

$$
F_{k}\left(X_{k} ; Y_{k} ; Z_{k}\right)=\mathbb{E}\left\{\prod_{\imath=1}^{k} G\left(x_{i}, y_{i} ; z_{\imath}\right)\right\},
$$

i.e. assuming that the complex spectral parameter in the Green functions are different. Indeed, to prove this assertion we only need to know that $\left|\operatorname{Im} z_{i}\right| \geq \eta_{1}$ for all $i=1, \ldots, k$ and to apply the analytic continuation arguments in the analog of Corollary 4.1 to each variable $z_{i}$ separately.

(2) We used $n^{-1} \sum_{\alpha=1}^{n} G(\alpha, x ; \alpha, y)$ as the basic quantity in the case of $a=n$ mainly because it is the Stieltjes transform of the respective IDS. We can however consider the Green function $G(\alpha, x ; \beta, y)$ itself. Then, adding in Definition (3.1) of the norm for $a=n$ the supremum with respect to all indices $\alpha_{1}, \ldots, \alpha_{k}, \beta_{1}, \ldots, \beta_{k}$, we can prove that in this norm the product

$$
\mathbb{E}\left\{\prod_{i=1}^{k} G\left(\alpha_{i}, x_{i} ; \beta_{i}, y_{i}\right)\right\}
$$

converges as $n \rightarrow \infty$ to

$$
\prod_{i=1}^{k} \delta_{\alpha_{i}, \beta_{i}} \mathscr{G}\left(x_{i}-y_{i}\right)
$$

(3) One may inquire to what extent our norms (3.1) are optimal. It is easy to see that they cannot be much stronger. Indeed, if the corresponding norm were strong enough to allow say the limiting transition $R \rightarrow \infty$ under the sum over $t$ in

$$
\mathbb{E}\left\{\sum_{t} G\left(x, t ; z_{1}\right) G\left(t, x ; z_{2}\right)\right\},
$$

then, according to (4.2) and (4.3), the limiting form of (4.32) would be

$$
F\left(z_{1}, z_{2}\right) \equiv \int_{\mathbb{T}^{d}}\left(\tilde{h}(k)-\zeta_{1}\right)^{-1}\left(\tilde{h}(k)-\zeta_{2}\right)^{-1} d k=\frac{r\left(\zeta_{1}\right)-r\left(\zeta_{2}\right)}{\zeta_{1}-\zeta_{2}},
$$

where $\zeta_{1,2}=\zeta\left(z_{1,2}\right), \zeta(z)=z+w^{2} r(z)$. On the other hand, according to the Hilbert identity and (4.3), the limiting form of (4.32) is $\left(r\left(z_{1}\right)-r\left(z_{2}\right)\right) /\left(z_{1}-z_{2}\right)$. Comparing this form with (4.33) we would conclude that $\zeta_{1}-\zeta_{2}=z_{1}-z_{2}$ for all $\left|\operatorname{Im} z_{1}\right|$, $\left|\operatorname{Im} z_{2}\right| \neq 0$, and we would obtain a contradiction to our main Eq. (4.5).

(4) However, in some cases we can improve the rate of convergence in Theorem 4.1. Consider, for instance the case of $a=n$ and assume that the function $h(x)$ in (2.3a) 
has a compact support. Then, by iterating the resolvent identity (3.8) with $H^{(n)}$ as $A+B$ and the nonrandom part of $H^{(n)}$ as $B$, we find that

$$
|G(\alpha, x ; \beta, y)| \leq C \exp \{-m|x-y|\},
$$

where $C$ and $\mathrm{m}$ are independent of $n$ and are strictly positive and finite for $0<|\operatorname{Im} z|<\infty$. Based on (4.34) and using an argument similar to that in the proof of Theorem 4.1, we can prove that

$$
\begin{aligned}
& \lim _{R \rightarrow \infty} \sup _{|\operatorname{Im} z| \geq \eta_{1}} \sup _{\left\{\alpha_{\imath}, \beta_{\imath}\right\}} \sup _{\left\{x_{\imath}, y_{\imath}\right\}} \exp \left\{m_{1} \sum_{i=1}^{k}\left|x_{i}-y_{i}\right|\right\} \\
& \quad \times\left|\mathbb{E}\left\{\prod_{i=1}^{k} G\left(\alpha_{i}, x_{\imath} ; \beta_{\imath}, y_{i}\right)\right\}-\prod_{\imath=1}^{k} \delta_{\alpha_{i}, \beta_{i}} \mathscr{G}\left(x_{i}-y_{i}\right)\right|=0
\end{aligned}
$$

for some $m_{1}<m$.

(5) According (4.2) and (4.3), the matrix $w^{2} \chi_{a} r_{a}(z) \delta(x-y)$ plays the role of the selfenergy operator $\Delta(z)=\{\Delta(x, y ; z)\}_{x, y \in \mathbb{Z}^{d}}$. The general definition of this operator is [21] $\mathbb{E}\{G(x, y ; z)\}=(h-z-\Delta(z))^{-1}(x, y)$. Thus, in our case this operator is diagonal and its site-independent diagonal entries are $w^{2} \lim _{a \rightarrow \infty} \mathbb{E}\left\{G_{a}(x, x ; z)\right\}$.

\section{The Deformed Semicircle Law}

Theorem 5.1. Let $H_{a}, a=R, n, d$ be the nonrandom operators defined in (2.1)-(2.3), $N_{0}(d \lambda)$ be defined by (2.12) for $a=R, n$ and by (2.16) for $a=d, N_{a}(d \lambda)$ be the IDS of $H_{a}$ given by (2.4) and (2.5) and $N_{a}(\lambda) \equiv N_{a}((-\infty, \lambda])$. Then for each $\lambda \in \mathbb{R}$,

$$
\lim _{a \rightarrow \infty} N_{a}(\lambda)=N(\lambda)
$$

where the Stieltjes transform (2.14) of $N(d \lambda)$ can be found as a unique solution of (2.15) in the class (2.10).

Proof. According to the spectral theorem, (2.4), (2.5) and (3.2), the Stieltjes transform of $N_{a}(d \lambda)$ is $\mathbb{E}\left\{G_{a}(0,0 ; z)\right\}$ and, according to Corollary 4.1 , for $k=1$,

$$
\lim _{a \rightarrow \infty}\left[\mathbb{E}\left\{G_{a}(0,0, z)\right\}-r_{a}(z)\right]=0,
$$

where $\operatorname{Im} z \neq 0$ and $r_{a}(z)$ is a unique solution of (4.5). By Lemma 4.2, we can perform the limiting transition $a \rightarrow \infty$, which replaces $\chi_{R}$ of (4.4) by unity (see (2.1c)) and $N_{0}(d \mu)$ of the form (2.12), (2.13) by (2.16) in the case of $a=d$. Thus, we have proved that the Stieltjes transform of $N_{a}(d \lambda)$ converges for $\operatorname{Im} z \neq 0$ to the solution of (2.15). Since this convergence implies weak convergence of the respective measures, we have thus proved weak convergence of $N_{a}(d \lambda)$ to $N(d \lambda)$. According to the property (5.6) below, $N(d \lambda)$ possesses a bounded density for any $N_{0}(d \lambda)$. This proves pointwise convergence in (5.1). The theorem is proved.

The limiting eigenvalue distribution defined by Eq. (2.15) is known as the deformed semicircle law. This distribution was found in [24] as the solution of a somewhat different problem than we are studying in this paper. Namely, let us consider a $l \times l$, $l=2 m+1$ random symmetric matrix

$$
h_{i}^{(l)} \delta_{i k}+l^{-1 / 2} W_{i k}^{(l)}, \quad|i|,|k| \leq m
$$


where a sequence $h_{\imath}^{(l)},|i| \leq m$ possesses the limiting distribution

$$
N_{0}(d \lambda)=\lim _{l \rightarrow \infty} l^{-1} \#\left\{h_{i}^{(l)} \in d \lambda\right\}
$$

and $W_{i k}^{(l)}$ are independent identically (for $i \leq k$ ) distributed random variables such that $[\mathrm{cf} .(2.1 \mathrm{e}, \mathrm{f})]$

$$
\begin{aligned}
\mathbb{E}\left\{W_{i k}^{(l)}\right\} & =0, \\
\mathbb{E}\left\{W_{i k}^{(l)} W_{j l}^{(l)}\right\} & =w^{2}\left[\delta_{i j} \delta_{k l}+\delta_{i l} \delta_{j k}\right] .
\end{aligned}
$$

Denote by $\lambda_{-m}^{(l)} \leq \ldots \leq \lambda_{m}^{(l)}$ the eigenvalues of the random matrix (5.2) and define the normalized counting function of this random matrix ensemble as [cf. (2.27)]

$$
N_{l}(\lambda)=l^{-1} \#\left\{\lambda_{l}^{(l)} \leq \lambda\right\} .
$$

Then, according to [24], there exists a nonrandom nondecreasing function $N(\lambda)$ called the integrated density of states of (5.2) and such that for each $\lambda \in \mathbb{R}$,

$$
p-\lim _{l \rightarrow \infty} N_{l}(\lambda)=N(\lambda)
$$

and the Stieltjes transform of $N(\lambda)$ satisfies (2.10) and (2.15).

In fact, the deformed semicircle law is the limiting eigenvalue distribution for (5.2) even for a more general case [24,10], when instead of identically distributed $W$ 's we consider independent random variables that satisfy some natural analog of the Lindeberg condition well known in probability theory. According to [10], this condition is also necessary if $h_{i}^{(l)}=0$. This case is known as the Wigner ensemble and the respective eigenvalue distribution is called the semicircle of Wigner law [see formula (5.5) below]. Recently, by using the method rather similar to that developed above we proved [16] that the deformed semicircle law is the limiting eigenvalue distribution in the general case.

$$
h_{\imath k}^{(l)}+l^{-1 / 2} W_{i k}^{(l)}, \quad|i|,|k| \leq m
$$

of the nondiagonal "nonperturbed" part having the limiting eigenvalue distribution $N_{0}(d \lambda)$ and of the Lindeberg's $W_{\imath k}^{(l)}$ 's. We also considered some other forms of random part in (5.2), in particular the so-called band random matrices [17] and diluted random matrices [18].

The simplest case of Eq. (2.15) corresponds to $N(d \lambda)=\delta(\lambda)$, when the unperturbed (nonrandom) part of (2.1)-(2.3) of (5.2) is zero. Then, $r=-\left(z+w^{2} r\right)^{-1}$ and

$$
r=\left(2 w^{2}\right)^{-1}\left(\left(z^{2}-4 w^{2}\right)^{-1 / 2}-z\right),
$$

where we use the branch of the radical that has a positive imaginary part on the upper edge of the cut $\operatorname{Im} z=0,|\operatorname{Re} z| \leq 2 w$. Equations (5.5) and (2.11) yield $N(d \lambda)=\varrho(\lambda) d \lambda$, where

$$
\varrho(\lambda)=\left(2 \pi w^{2}\right)^{-1} \begin{cases}\left(4 w^{2}-\lambda^{2}\right)^{-1 / 2}, & |\lambda| \leq 2 w \\ 0, & |\lambda|>2 w .\end{cases}
$$

This is the well known Wigner or semicircle law (see [22, 23, 32] for the references, the history and numerous related results).

Now we will prove some useful properties of the deformed semicircle law, defined by Eq. (2.15). 
(i) For any nonperturbed $N_{0}(d \lambda)$,

$$
N(d \lambda)=\varrho(\lambda) d \lambda, \quad 0 \leq \varrho(\lambda) \leq(\pi w)^{-1} .
$$

Proof. For $r_{2}(\lambda+i \eta) \equiv \operatorname{Im} r(\lambda+i \eta)$ (2.15) yields $r_{2} \leq\left(\eta+w^{2} r_{2}\right)^{-1}$. Thus, $r_{2}(\lambda+i \eta) \leq w^{-1}$. This inequality and the inversion formula (2.11) imply (5.7).

(ii) Let us call $\operatorname{supp} N$ and $\operatorname{supp} N_{0}$ the spectrum $\sigma$ and the unperturbed spectrum $\sigma_{0}$. Then, $\sigma$ is contained in the $2 w$-neighbourhood of $\sigma_{0}$.

Proof. If

$$
I(\lambda, \eta) \equiv \int\left|\mu-z-w^{2} r(z)\right|^{-2} N_{0}(d \lambda), \quad z=\lambda+i \eta
$$

and $r_{2} \geq 0$ is as above, then

$$
I=r_{2}\left(\eta+w^{2} r_{2}\right)^{-1} \leq w^{-2} .
$$

According to (2.15) and the Schwarz inequality, $|r(z)| \leq I^{1 / 2} \leq w^{-1}$. If $\lambda$ is outside of the $2 w$-neighbourhood of $\sigma_{0}$, then $\left|\mu-\lambda-w^{2} r(\lambda+i 0)\right|>w$, and since $N_{0}(\mathbb{R})=1$, then we find from $(5.7)$ that $I(\lambda, 0)<w^{-2}$. On the other hand any open interval intersecting with $\sigma$ contains points at which $r_{2}(\lambda+i 0)>0$. For these points, according to $(5.8), I(\lambda, 0)=w^{2}$. This contradiction proves our assertion.

(iii) If $a$ and $b$ are the left and right endpoints of the interval containing $\sigma$ and $a_{0}$ and $b_{0}$ are the same points for $\sigma_{0}$, then $a<a_{0}$ and $b>b_{0}$.

Proof. If $\lambda>b$, then $r_{2}(\lambda+i 0)=0, r(\lambda)=r(\lambda+i 0)$ is a nonpositive strictly increasing function, and if $\lambda$ varies from $b+0$ to $\infty$, then $r(\lambda)$ varies from $r(b+0)<0$ to 0 . This and (2.15) imply that the interval $(r(b+0), \infty)$ does not intersect with $\sigma_{0}$, i.e. $b_{0} \leq b+r(b+0)$ and $b-b_{0} \geq-r(b+0)>0$. The inequality $a<a_{0}$ is proved similarly.

Till now we have not used the fact that, according to (2.12) and (2.13), $\sigma_{0}$ is an interval, i.e. $\sigma_{0}=\left(a_{0}, b_{0}\right)$.

(iv) If $\sigma_{0}=\left(a_{0}, b_{0}\right)$, then $\sigma=(a, b)$ (and according to (ii) and (iii), $\left(a_{0}, b_{0}\right) \subset(a, b) \subset$ $\left.\left(a_{0}-2 w, b_{0}+2 w\right)\right)$.

Proof. Assume that some interval $(\alpha, \beta) \subset(a, b)$ does not belong to $\sigma$. Then it does not belong to $\sigma_{0}$. Indeed, in the opposite case, some interval $\left(\alpha_{1}, \beta_{1}\right)=(\alpha, \beta) \cap \sigma_{0}$ is not empty and its image under the continuous map $\lambda+r(\lambda)$ does not intersect with $\left(a_{0}, b_{0}\right)$. Thus, either $\beta_{1}+r\left(\beta_{1}\right)<a_{0}$ or $\alpha_{1}+r\left(\alpha_{1}\right)>b_{0}$. But, according to (2.15), in the former case, $r\left(\beta_{1}\right)>0$ and $\beta_{1}<a_{0}$, and in the latter case, $r\left(\alpha_{1}\right)<0$ and $\alpha_{1}>b_{0}$. This means that $\left(\alpha_{1}, \beta_{1}\right)$ does not belong to $\left(a_{0}, b_{0}\right)$.

Let us show that $(a, b) \backslash\left(a_{0}, b_{0}\right)$ belongs to $\sigma$. Assume first that $\lambda>b>b_{0}$. Then $r(\lambda) \equiv r(\lambda+i 0)$ and $r_{0}(\lambda) \equiv r_{0}(\lambda+i 0)$ are nonpositive strictly decreasing functions and both have strictly increasing inverse functions $\lambda(r)$ and $\lambda_{0}(r)$, that, according to (2.15), satisfy the relation

$$
\lambda(r)=\lambda_{0}(r)-w^{2} r .
$$

If $r^{*}$ is specified as $\lambda_{0}^{\prime}\left(r^{*}\right)=w^{2}$ then $b^{*} \equiv \lambda_{0}\left(r^{*}\right)-w^{2} r^{*} \geq b$. Let us assume now that $b_{0}<\alpha, \beta<b^{*}$ and $(\alpha, \beta)$ does not belong to $\sigma$. Then again $\lambda(r)$ and $\lambda_{0}(r)$ are well defined, strictly decreasing, satisfy (5.10) and its 1.h.s. assumes values strictly 
less than $b^{*}$. But we have just showed that the r.h.s. of (5.10) does not assume values less than $b^{*}$. Thus $b^{*}=b$.

The same arguments show that $\left(a, a_{0}\right)$ belongs to $\sigma$ and that $a$ can be defined as $\lambda_{0}(a)=w^{2}, a<a_{0}$.

In fact, we have proved a rule to determine $\sigma$.

(v) Consider the intervals that comprise the complement of $\sigma_{0}$, find the inverse $\lambda_{0}(r)$ to $r_{0}(\lambda)$ for these intervals, locate the intervals on which the function $\lambda_{0}(r)-w^{2} r$ is monotonically increased and then determine the set of values of this function on these intervals. The spectrum $\sigma$, is the complement of this set.

If $\alpha_{0}$ is an endpoint of one of the intervals, then $a=\lambda_{0}(\alpha)-w^{2} \alpha$ is the endpoint of one of the components of $\sigma$. Suppose that in the neighbourhood of $\alpha, \lambda_{0}(r)-w^{2} r$ is analytic. Then simple arguments show that the density of states in the neighbourhood of $\alpha$ behaves as follows:

$$
\varrho(\lambda)=\text { const }|\lambda-a|^{1 / 2 k}(1+o(1)), \quad|\lambda-a| \rightarrow 0
$$

for some $k=1,2, \ldots$. Generically, $k=1$, and this corresponds to the nondegenerated extremum of $\lambda_{0}(r)-w^{2} r$ (a maximum if $\alpha$ is the right endpoint, or a minimum if $\alpha$ is the left endpoint of such an interval).

\section{The Conductivity}

As was explained in Sect. 2, we are going to prove weak convergence of the measures (2.24)-(2.26) for $\alpha \rightarrow \infty$ and calculate the respective limits. Therefore it suffices to consider their Stieltjes transforms

$$
C^{(a)}\left(z_{1}, z_{2}\right)=\int \frac{M_{(a)}\left(d \lambda_{1}, d \lambda_{2}\right)}{\left(\lambda_{1}-z_{1}\right)\left(\lambda_{2}-z_{2}\right)}, \quad \operatorname{Im} z_{1,2} \neq 0
$$

and find their limits for $a \rightarrow \infty$ for $\operatorname{Im} z_{1,2} \neq 0$. After that we can apply the inversion formula (2.11) to each of the two variables and find the limiting measure. Calculation of (6.1) for $a \rightarrow \infty$ includes a considerable amount of technical material. For this reason we will consider here the simplest case of the Gaussian-distributed randomness in (2.1)-(2.3), postponing the proof for an arbitrarily distributed randomness for a subsequent publication.

Now it is clear that our results (2.29) are equivalent to

Theorem 6.1. Let $H(a), a=R, d, n$ be given by (2.1)-(2.3) in in which

$$
\begin{gathered}
h(x)=h(-x), \\
\sum_{x \in \mathbb{Z}^{d}}|x||h(x)|<\infty
\end{gathered}
$$

and $W$ 's be the Gaussian-distributed random variables. Then, for $\operatorname{Im} z_{1,2} \neq 0$,

$$
\lim _{\alpha \rightarrow \infty} C^{(a)}\left(z_{1}, z_{2}\right)= \begin{cases}r\left(z_{1}\right) r\left(z_{2}\right) \int x^{2} \phi^{2}(x) d x, & a=R \\ r\left(z_{1}\right) r\left(z_{2}\right), & a=d \\ \int \frac{|\nabla \tilde{h}(k)|^{2} d k}{\left(\tilde{h}(k)-z_{1}-w^{2} r\left(z_{1}\right)\right)\left(\tilde{h}(k)-z_{2}-w^{2} r\left(z_{2}\right)\right)}, & a=n,\end{cases}
$$


where $r(z)$ is given by (2.15) and (2.12) for $a=R, n$ and by (2.15) and (2.17) for $a=n$.

Proof. Since the proof of (6.4) is somewhat tedious even for the Gaussian $W$ 's in (2.1)-(2.3), we describe in detail the case of $a=n$ that turns out to be simplest and after that explain additional steps needed in the other cases $(a=R, d)$. Note also that the case $a=n$ was considered for the first time in [31] where the results (2.29) and (6.4) were obtained by the perturbation theory arguments.

According to (2.3) and (2.20), the velocity operator in this case is identity with respect to the orbital indices $\alpha, \beta$ and its coordinate-dependent part is defined by the matrix

$$
\hat{v}^{(n)}(x-y)=i(x-y) h(x-y)
$$

and is bounded in view of (6.3). Therefore, according to (6.1) and (6.5),

$$
C^{(n)}\left(z_{1}, z_{2}\right)=\sum_{y, t} \sum_{i=1}^{d} \hat{v}_{i}(y) \hat{v}_{i}(t) T_{0}\left(0, y, t ; z_{1}, z_{2}\right)
$$

where

$$
T_{0}\left(x, y, t ; z_{1}, z_{2}\right)=\mathbb{E}\left\{n^{-1} \sum_{\alpha=1}^{n} Q\left(x, y, t, z_{1}, z_{2}, \alpha, \alpha\right)\right\}
$$

and

$$
Q\left(x, y, t, z_{1}, z_{2}, \alpha, \gamma\right)=\sum_{\beta, u} G\left(\alpha, x ; \beta, u+t ; z_{1}\right) G\left(\beta, u ; \gamma, y ; z_{2}\right)
$$

Consider now the infinite sequence of moments containing (6.7)

$$
\begin{aligned}
& T_{k}\left(X_{k} ; Y_{k} ; Z_{k} ; x, y, t, z_{1}, z_{2}\right) \\
& \quad=\mathbb{E}\left\{\prod_{j=1}^{k} G\left(x_{j}, y_{j} ; \zeta_{j}\right) n^{-1} \sum_{\alpha=1}^{n} Q\left(x, y, t, z_{1}, z_{2}, \alpha, \alpha\right)\right\}, \quad k \geq 0
\end{aligned}
$$

where $G(x, y ; \zeta)$ is specified by (3.2) for $a=n$ and $z=\zeta,\left|\operatorname{Im} \zeta_{i}\right| \geq \eta,\left|\operatorname{Im} z_{1,2}\right| \geq \eta$.

Now, by using the resolvent identity for $G\left(\beta, u ; \alpha, y ; z_{2}\right)$ and the arguments similar to those in the derivation of (3.7), we obtain the system of relations

$$
\begin{aligned}
& T_{k}\left(X_{k} ; Y_{k} ; Z_{k} ; x, y, t, z_{1}, z_{2}\right)=\sum_{u} F_{k+1}\left(X_{k}, x ; Y_{k},(u+t) ; Z_{k}, z_{1}\right) g_{2}(u-y) \\
& \quad+w^{2} \sum_{s}\left[T_{k+1}\left(X_{k}, x ; Y_{k}, s ; Z_{k}, z_{1} ; s, s, t, z_{1}, z_{2}\right)\right. \\
& \left.\quad+T_{k+1}\left(X_{k}, s ; Y_{k}, s ; Z_{k}, z_{2} ; x, s, t, z_{1}, z_{2}\right)\right] g_{2}(s-y)+R_{k}
\end{aligned}
$$

where $g_{2}(x-y)=\left(h-z_{2}\right)^{-1}(x-y)$ and

$$
R_{k}=R_{k}^{(1)}+R_{k}^{(2)}+R_{k}^{(3)}
$$


with

$$
\begin{aligned}
R_{k}^{(1)}= & w^{2} n^{-2} \sum_{j=1}^{k} \sum_{s, \alpha, \gamma} \mathbb{E}\left\{G_{1} \ldots G_{\jmath-1}\right. \\
& \times\left[G\left(\alpha, x_{j} ; \gamma, s\right) G\left(\alpha, s ; \alpha, y_{\jmath}\right)+G\left(\gamma, x_{j} ; \alpha, s\right) G\left(\gamma, s ; \alpha, y_{j}\right)\right] \\
& \left.\times G_{\jmath+1} \ldots G_{k} Q(x, s, t, \alpha, \gamma)\right\} g_{2}(s-y), \\
R_{k}^{(2)}= & w^{2} n^{-2} \sum_{s, \alpha, \gamma} \mathbb{E}\left\{\prod_{j=1}^{k} G_{j} G\left(\alpha, x ; \gamma, s, z_{1}\right) Q(s, s, t, \alpha, \gamma)\right\} g_{2}(s-y), \\
R_{k}^{(3)}= & w^{2} n^{-2} \sum_{s, \alpha, \gamma} \mathbb{E}\left\{\prod_{\jmath=1}^{k} G_{j} G\left(\alpha, s ; \gamma, s, z_{2}\right) Q(x, s, t, \alpha, \gamma)\right\} g_{2}(s-y),
\end{aligned}
$$

where $G_{j} \equiv G\left(x_{j}, y_{j} ; \zeta_{j}\right)$.

Arguments based on (2.3c), (3.28) and (3.29) and similar to those used in obtaining the bound (3.24) for (3.20) yield for $\eta>2 \bar{h}$ (see (3.12))

$$
\begin{aligned}
|Q(x, s, t, \alpha, \gamma)| & \leq\left[\sum_{\beta, u}\left|G_{1}(\alpha, x ; \beta, u+t)\right|^{2} \sum_{\beta, u}\left|g_{2}(\beta, u ; \gamma, y)\right|^{2}\right]^{1 / 2} \\
\left|R_{k}^{(1)}\right| & \leq 4 n^{-1} w^{2} k \eta^{-k-4} \\
\left|R_{k}^{(2)}\right| & \leq 2 n^{-1} w^{2} k \eta^{-k-4}
\end{aligned}
$$

and the same bound for $R_{k}^{(3)}$. As a result,

$$
\left|R_{k}\right| \leq 4 n^{-1} w^{2}(k+1) \eta^{-k-4} .
$$

Consider now the Banach space of the sequences

$$
T=\left\{T_{k}\left(X_{k} ; Y_{k} ; Z_{k} ; x, y, t, z_{1}, z_{2}\right)\right\}_{k=0}^{\infty}
$$

with the norm

$$
\|T\|=\sup _{k \geq 0} \xi^{k} \sup _{\left|\operatorname{Im} \zeta_{k}\right|,\left|\operatorname{Im} z_{1,2}\right| \geq \eta} \sup _{X_{k}, Y_{k}, x, y, t}\left|T_{k}\right| .
$$

If $A_{1}$ is the linear operator defined by the sum over $s$ in $(6.10)$, then it is easy to show that

$$
\left\|\mathscr{C}_{1}\right\| \leq 4 w^{2} \xi^{-1} \eta^{-1}
$$

and if $R$ is the sequence specified by (6.11)-(6.14), then in view of (6.17),

$$
\|R\| \leq 4 n^{-1} w^{2} \eta^{-4} \sup _{k \geq 0} k\left(\xi \eta^{-1}\right)^{k}
$$

Thus, to guarantee a finite norm of $T$ in (6.18), the contractivity property of $A_{1}$ and the finiteness of $\|R\|$, it suffices to take

$$
\xi=\eta / 2, \quad \eta=\max \{3 w, 2 \bar{h}\} .
$$

Then,

$$
\left\|\mathscr{C}_{1}\right\| \leq \frac{8}{9}<1, \quad\|R\| \leq C n^{-1}
$$


where $C$ is independent of $n$. If, in addition, $\bar{F}=\left\{\bar{F}_{k}\right\}_{k \geq 0}$ is the sequence defined by the first sum in the r.h.s. of (6.10), then

$$
\|\bar{F}\| \leq \eta^{-2} \sup _{k \geq 1}\left(\xi \eta^{-1}\right)^{k}<\infty .
$$

Theorem 4.1 and summability of $g_{2}(x)$ [see (3.12)] imply that

$$
\bar{F}=\overline{\mathscr{G}}+\varepsilon_{n},
$$

where $\lim _{n \rightarrow \infty}\left\|\varepsilon_{n}\right\|=0$ and $\overline{\mathscr{G}}=\left\{\overline{\mathscr{G}}_{k}\right\}_{k=0}^{\infty}$ with

$\overline{\mathscr{G}}_{k}\left(X_{k} ; Y_{k} ; Z_{k} ; x, y, t, z_{1}, z_{2}\right)=\prod_{j=1}^{k} \mathscr{G}\left(x_{\jmath}-y_{j} ; \zeta_{j}\right) \sum_{u} \mathscr{G}\left(x-u-t ; z_{1}\right) g\left(u-y ; z_{2}\right)$,

where $\mathscr{G}(x ; z)$ is specified by $(4.3)-(4.5)$.

Now, if $\bar{T}=\left\{\bar{T}_{k}\right\}_{k=0}^{\infty}$ is a unique solution of the equation

$$
\bar{T}=\overline{\mathscr{G}}+\mathscr{b}_{1} \bar{T}
$$

then

$$
\lim _{n \rightarrow \infty}\|T-\bar{T}\|=0
$$

and $\bar{T}_{k}\left(X_{k} ; Y_{k} ; Z_{k} ; x, y, t, z_{1}, z_{2}\right)=\prod_{j=1}^{k} \mathscr{G}\left(x_{j}-y_{j} ; \zeta_{j}\right) \overline{\mathscr{G}}\left(x-y, t ; z_{1}, z_{2}\right)$, here $\mathscr{G}\left(x, t ; z_{1}, z_{2}\right)$ is a solution of the equation

$$
\begin{aligned}
\overline{\mathscr{G}}(x-y, t)= & \sum_{u} \mathscr{\mathscr { S }}\left(x-t-u ; z_{1}\right) g\left(u-y ; z_{2}\right) \\
& +w^{2} \sum_{s}\left[\mathscr{\mathscr { G }}\left(0 ; z_{2}\right) \overline{\mathscr{G}}(x-s, t)+\mathscr{G}\left(x-s ; z_{2}\right) \overline{\mathscr{G}}(0, t)\right],
\end{aligned}
$$

provided that this equation is uniquely solvable. The latter fact can readily be proved in the space with the norm

$$
\|\overline{\mathscr{G}}\|=\sup _{\left|\operatorname{Im} z_{1,2}\right| \geq \eta} \sup _{x, t}\left|\mathscr{G}\left(x, t ; z_{1}, z_{2}\right)\right|
$$

with $\eta$ specified by (6.22). Besides, it is easy to check by direct calculation that

$$
\overline{\mathscr{G}}\left(x, t ; z_{1}, z_{2}\right)=\mathscr{G}_{12}(x+t)+w^{2} \mathscr{G}_{12}(x) \mathscr{G}_{12}(t)\left(1-\mathscr{G}_{12}(0)\right)^{-1},
$$

where $\mathscr{G}_{12}(x-t)$ is the product of the Toeplitz operators $\left.\mathscr{G}\right|_{z=z_{1,2}}$ whose kernel is specified by (4.3), i.e.

$$
\mathscr{G}_{12}(x)=\int_{\mathbb{T}^{d}} \frac{\exp (2 \pi i k x) d k}{\left(\tilde{h}(k)-z_{1}-w^{2} r\left(z_{1}\right)\right)\left(\tilde{h}(k)-z_{2}-w^{2} r\left(z_{2}\right)\right)} .
$$

According to (6.2), $\tilde{h}(k)$ is even. Thus, $\mathscr{G}_{12}(x)$ has the same property and since $\hat{v}(x)$ specified by (6.5) is odd, the second term of (6.27) gives no contribution to (6.6). Besides, since the Fourier transform of $\hat{v}(x)$ is $\nabla \tilde{h}(k)$, the substitution of (6.26) into (6.6) yields (6.4) for $a=n$ and $\left|\operatorname{Im} z_{1,2}\right| \geq \eta$. By using the analytic continuation 
arguments it is easy to show that the same limiting relation is true for all $\operatorname{Im} z_{1,2} \neq 0$. Thus, we have proved the proposition for $a=n$.

According to (2.24) and (6.1), in the case of $a=R$,

$$
C^{(R)}\left(z_{1}, z_{2}\right)=R^{-2} \sum_{i=1}^{d} \mathbb{E}\left\{\hat{v}_{i} G\left(z_{1}\right) \hat{v}_{i} G\left(z_{2}\right)\right\},
$$

and without loss of generality we can set $h(x) \equiv 0$ in (2.1). However, in this case the velocity operator (2.20) is random:

$$
\hat{v}^{(R)}(x, y)=R^{-d / 2}(x-y) \phi((x-y) / R) W(x, y),
$$

and, as a result, the expression under the expectation sign in (6.29) is bilinear in $W$ 's. Calculating this expectation with the help of (3.13) and (3.14), we find the term

$$
R^{-d-2} \sum_{x}|x|^{2} \phi^{2}(x / R)\left[\mathbb{E}\left\{G_{1}(0,0) G_{2}(x, x)+G_{1}(0, x) G_{2}(0, x)\right\}\right],
$$

which results from differentiating one $W$ with respect to another $W$ and twelve terms due to differentiating the Green functions with respect to $W$ 's.

According to Theorem 4.1, the first term in (6.30) converges to

$$
\overline{x^{2}} r\left(z_{1}\right) r\left(z_{2}\right), \quad \overline{x^{2}} \equiv \int_{\mathbb{R}^{d}} x^{2} \phi(x) d x,
$$

while the second term in (6.28) vanishes, because it is bounded by

$$
\phi_{0}^{2} R^{-d} \mathbb{E}\left\{\sum_{x}\left|G_{1}(0, x) G_{2}(0, x)\right|\right\} \leq \phi_{0}^{2} R^{-d} \eta^{-2} .
$$

Similar but more cumbersome calculations allow us to show that among the twelve terms only the following four do not vanish in the limit $R=\infty$ :

$$
w^{2} R^{-2} \mathbb{E}\left\{\left[\Delta_{1} G_{1} \Delta_{1} G_{2}+G_{1} \Delta_{2} G_{2} \Delta_{2}+\Delta_{1} G_{1} \Delta_{2} G_{2}+G_{1} \Delta_{1} G_{2} \Delta_{2}\right](0,0)\right\},
$$

where $G_{1,2}=\left.G(z)\right|_{z=z_{1,2}}$ and $\Delta_{1,2}$ are the diagonal operators with the entries

$$
\Delta_{i}(x)=R^{-d} \sum_{y}(x-y) \phi^{2}((x-y) / R) G_{i}(y, y) .
$$

To calculate the $R=\infty$ limit of each term in (6.32), we again derive the infinite system of relations for the infinite family of the Green function moments that includes this term. This system, just as system (3.5) and (6.10), is asymptotically close to the system of equations that is similar to (4.16) and (6.25) and is explicitly soluble. The role of the free term in the latter is played by the expression

$$
R^{-2 d-2} \sum_{i=1}^{d} \sum_{x, y} x_{i} y_{i} \phi^{2}(x / R) \phi^{2}(y / R) \mathbb{E}\left\{G_{1}(x, x) G_{2}(y, y)\right\}
$$

which, according to Theorem 4.1 has the limit

$$
r\left(z_{1}\right) r\left(z_{2}\right) \sum_{i=1}^{d}\left[\int x_{i} \phi^{2}(x) d x\right]^{2} .
$$


To guarantee symmetry of the matrix $(2.1), \phi(x)$ has to be even. Thus, the integrals in (6.34) vanish. This means that the $R=\infty$ limits of all terms in (6.32) are equal to zero and we arrived at expression (6.31) for $\lim _{R \rightarrow \infty} C^{(R)}\left(z_{1}, z_{2}\right)$.

The case of $a=d$ is similar, the role of $\phi(x / R)$ is played by $\delta(x-1)$ (see (3.6)). This results in replacement of $\overline{x^{2}}$ by 1 in (6.31) for $a=d$. The proposition is proved.

We have seen that in the case of $a=R, d$, the proof is rather lengthy and the final fairly simple result emerges only at the last stage, due to the zero value of (6.34). Thus, it would be of some interest to discuss another strategy of proof which is simpler at least conceptually. This strategy is based on another formula for the conductivity. To explain this formula, it is convenient to begin with the prelimit form of the measure (2.24) in the finite volume [8, 21]. For $a=R$ this measure is [cf. (2.28)]

$$
M_{\Lambda}^{(R)}\left(d \lambda_{1}, d \lambda_{2}\right)=R^{-2}|\Lambda|^{-1} \sum_{\imath=1}^{d} \operatorname{Tr}\left[\mathscr{E}_{\Lambda}\left(d \lambda_{1}\right) \hat{v}_{\imath} \mathscr{E}_{\Lambda}\left(d \lambda_{2}\right) \hat{v}_{\imath}\right]
$$

By using the spectral expansion for $H_{\Lambda}^{(R)}$, we obtain

$$
\begin{aligned}
M_{\Lambda}^{(R)}\left(d \lambda_{1}, d \lambda_{2}\right)= & (2 R)^{-2}\left(\lambda_{1}-\lambda_{2}\right)^{2}|\Lambda|^{-1} \\
& \times \sum_{x, y \in \Lambda}(x-y)^{2} \mathbb{E}\left\{\mathscr{E}_{\Lambda}\left(x, y ; d \lambda_{1}\right) \mathscr{E}_{\Lambda}\left(y, x ; d \lambda_{2}\right)\right\} .
\end{aligned}
$$

Thus, after performing the thermodynamic limit $\Lambda \rightarrow \infty$, we have

$$
M^{(R)}\left(d \lambda_{1}, d \lambda_{2}\right)=-2^{-1}\left(\lambda_{1}-\lambda_{2}\right)^{2} L^{(R)}\left(d \lambda_{1}, d \lambda_{2}\right)
$$

where

$$
L^{(R)}\left(d \lambda_{1}, d \lambda_{2}\right)=R^{-2} \sum_{t} t^{2} \mathbb{E}\left\{\mathscr{E}\left(0, t ; d \lambda_{1}\right) \mathscr{E}\left(t, 0 ; d \lambda_{2}\right)\right\}
$$

The Stieltjes transform of this measure is

$$
D\left(z_{1}, z_{2}\right)=R^{-2} \sum_{t} t^{2} \mathbb{E}\left\{G\left(0, t ; z_{1}\right) G\left(t, 0 ; z_{2}\right)\right\}
$$

It seems that (6.39) makes our problem quite simple. Indeed, in view of Theorem 4.1 the expectation in (6.39) is factorized at the limit $R=\infty$ into the product $\mathscr{G}\left(x ; z_{1}\right)$ $\mathscr{G}\left(-x ; z_{2}\right)$. However, it is easy to see that replacement of the expectation in (6.38) by this product results in an incorrect answer. The formal reason is that the norm (3.1) that we used in Proposition 3.1, does not allow us to interchange the sum over $t$ and the limiting transition $R \rightarrow \infty$. We have demonstrated in Remark 3 of Sect. 4 that this interchange is impossible even in the simpler expression (4.32). Therefore, we cannot use Theorem 4.1 in the asymptotic calculation of (6.38).

In fact, the question of interchangeability of summing over the whole space and the respective limiting transition should have been posed earlier, in the case of the expectations in (6.7) and (6.29). The answer should be the same as above, although in the case of $a=n$, due to the diagonality of the random part of (2.3a) with respect to the coordinates, the rate of convergence with respect to the coordinate (but not with respect to orbital indices $\alpha, \beta$, etc.) may be high enough (see e.g. (4.35)) to guarantee interchangeability of the sum over the coordinates (but not over the orbital indices) 
and the limiting transition $n \rightarrow \infty$. In particular, this was the case with the calculation of the $n=\infty$ limit of the static conductivity that was carried out by Wegner [31] and was based on $n$-component analogs of formulas (6.36) and (6.38), in which the $n$-component analog

$$
n^{-1} \sum_{\alpha, \beta=1}^{n} \mathbb{E}\left\{G\left(\alpha, 0 ; \beta, x ; z_{1}\right) G\left(\beta, x ; \alpha, 0 ; z_{2}\right)\right\}
$$

of the expectation in (6.38) was asymptotically calculated for a fixed $t$, i.e. inside the sum over $t$ in (6.39).

Thus, as we have seen above, we cannot reduce the asymptotic calculation of (6.38) for $R \rightarrow \infty$ to Theorem 4.1. We can however apply the method that was used to prove this theorem. Namely, we should find some infinite family of moments of $G$ 's which includes (6.38) and satisfies up to small terms an infinite system of explicitly soluble equations.

We consider the following family [cf. (6.9)]:

$$
\begin{aligned}
& D_{k}\left(X_{k} ; Y_{k} ; Z_{k} ; x, y, z_{1}, z_{2}\right) \\
& \quad=\mathbb{E}\left\{\prod_{j=1}^{k} G\left(x_{j}, y_{j} ; \zeta_{\jmath}\right) R^{-2} \sum_{u}(u-x)(u-y) G\left(x, u ; z_{1}\right) G\left(u, y ; z_{2}\right)\right\} .
\end{aligned}
$$

It is clear that $D_{0}\left(0,0, z_{1}, z_{2}\right)$ coincides with (6.39). By using the arguments similar to those in the derivation of (3.5) and (6.10), we find that

$$
\begin{aligned}
D_{k}\left(X_{k} ; Y_{k} ; Z_{k} ; x, y\right)= & \mathbb{E}\left\{\prod_{\jmath=1}^{k} G_{\jmath} R^{-2} \sum_{u}(u-x)(u-y) G\left(x, u ; z_{1}\right) g\left(u-y ; z_{2}\right)\right\} \\
& +w^{2} R^{-d} \sum_{s, t} \phi^{2}((s-t) / R) g\left(t-y ; z_{2}\right) \\
& \times\left[D_{k+1}\left(X_{k}, x ; Y_{k}, t ; Z_{k}, z_{1} ; s, s\right)\right. \\
& +D_{k+1}\left(X_{k}, s ; Y_{k}, s ; Z_{k}, z_{2} ; x, s\right) \\
& \left.+(s-x)(s-y) \mathbb{E}\left\{\prod_{j=1}^{k} G_{\jmath} \sum_{n} G\left(x, u ; z_{1}\right) G\left(u, y ; z_{2}\right)\right\}\right]+U_{k}
\end{aligned}
$$

where $U_{k}$ vanishes as $R \rightarrow \infty$ [cf. $S_{k}$ in (6.5) and $R_{k}$ in (6.10)]. Omitting this remainder, we can solve the resulting system of integral equations by the ansatz

$$
D_{k}\left(X_{k} ; Y_{k} ; Z_{k} ; x, y\right)=\prod_{j=1}^{k} \mathscr{G}\left(x_{\jmath}-y_{j} ; z_{j}\right) D_{0}(x-y)
$$


where $D_{0}(x)$ satisfies the equation

$$
\begin{aligned}
D_{0}(x-y)= & w^{2} R^{-d} \sum_{s, t} \phi^{2}((s-t) / R) g\left(t-y ; z_{2}\right) \\
& \times\left[D_{0}(x-t) \mathscr{G}\left(0 ; z_{2}\right)+D_{0}(0) \mathscr{G}\left(x-t ; z_{1}\right)\right. \\
& \left.+\mathscr{G}\left(x-y ; z_{1}\right)(s-x)(s-y) \sum_{u} \mathbb{E}\left\{G\left(x, u ; z_{1}\right) G\left(u, y ; z_{2}\right)\right\}\right] .
\end{aligned}
$$

By using the Hilbert identity and Theorem 4.1 to calculate the last term, we find that

$$
D_{0}(x-y)=\mathscr{G}_{1,2}(x-y) \overline{x^{2}} w^{2} F^{-1}\left(\frac{r\left(z_{1}\right)-r\left(z_{2}\right)}{z_{1}-z_{2}}\right)^{2},
$$

where $\mathscr{G}_{1,2}(x), \overline{x^{2}}$ and $F$ are defined in (6.28), (6.31) and (4.33), respectively. It is easy to show that (6.37)-(6.39) and (6.42) yield the same expression (6.4) for the density of the limiting measure.

The above method of calculation of this density seems more direct than that based on (2.19) and requiring a considerable amount of calculations. However, the rigorous justification of all the stages starting from substantiating the limiting form (6.37) of (6.36) is also rather cumbersome and the respective amount of calculations is practically the same.

We note in the conclusion that based on (6.42) we can calculate zero frequency conductivity $\sigma_{\mathrm{dc}}(E)=\sigma(0, E)$ in two ways. In the first one we just set $\nu=0$ in (2.22). In the second way we use the formula

$$
\sigma_{\mathrm{dc}}(E)=\lim _{\varepsilon \downarrow 0} \varepsilon^{2} \sum_{x \in \mathbb{Z}^{d}}|x|^{2} \mathbb{E}\left\{|G(0, x ; \lambda+i \varepsilon)|^{2}\right\},
$$

which is widely accepted in solid state physics and shows that direct current conductivity is physically related to diffusion. This formula is a version of the Kubo formula derived for the direct current.

Interchanging the limits $\varepsilon \downarrow 0$ and $R \rightarrow \infty$ in this formula we obtain the possibility to use (6.42) for $x=y, z_{1,2}=E \pm i \varepsilon$. Performing the limit $\varepsilon \downarrow 0$ in the respective expression, we again arrive at (2.29).

\section{Discussion}

As we mentioned in Sect. 5, the deformed semicircle law appeared for the first time [24] in a somewhat different problem on the limiting eigenvalue distribution of the random matrices (5.2) (see also (5.4)), known as the deformed Wigner ensemble. This ensemble arose in nuclear physics (see e.g. $[22,32]$ ), where it was proposed in order to describe the statistics of low-lying levels of heavy nuclei. Later similar ensembles appeared in quantum field theory [9], quantum chaology [11] and statistical mechanics [5].

The main difference of the respective random matrices (5.2) and (5.4) from those modelling elementary excitations in disordered condensed matter [in particular, the random operators (2.1)-(2.3)] is that the former have all the entries of the same order 
of magnitude (e.g. identically distributed), while the latter have nonzero entries only on the finite number $b$ of diagonals adjacent to the principal diagonal (for instance in (2.1) $b=(2 r R)^{d}+1$, where $r$ is the radius of the support of $\left.\phi(t)\right)$. In other words the former random matrices correspond to nonlocally interacting disordered systems (the range of the interaction is of the order of the size of the system), while the latter correspond to the locally interacting (short range) systems. Therefore it is rather natural that we have obtained the deformed semicircle law as the result of the limiting transition $R, d, n \rightarrow \infty$.

Let us consider the finite volume version of (2.1), i.e. the restriction $H_{\Lambda}^{(R)}$ of (2.1) to a finite cube $\Lambda \subset \mathbb{Z}^{d}$ centred at the origin and having the side length $L$. Then (5.4) corresponds to $d=1, L=\ell=2 m+1, R=2 m$ and $\phi(t)=\chi_{1}(t)$, where $\chi_{1}(t)$ is the indicator of the interval $[-1 / 2,1 / 2]$. Therefore Theorem 5.1 of this paper and the results of [24] (see also [16]) show that the deformed semicircle law is the limiting eigenvalue distribution in the two extreme cases of $H_{\Lambda}^{(R)}$ : the first one corresponds to the two successive limiting transitions $L \rightarrow \infty$ and then $R \rightarrow \infty$ (Theorem 5.1), while the second one corresponds to the simultaneous limits $L \rightarrow \infty, R \rightarrow \infty, L R^{-1} \rightarrow 1$. In view of these results it is natural to analyse the intermediate cases when $L \rightarrow \infty$ and $R \rightarrow \infty$ but $0 \leq \nu \equiv \lim L R^{-1} \leq 1$. It is the so-called band random matrices, which appear for instance in the studies of quantum chaos [6]. In paper [17] it was shown that under fairly general conditions (condition (5.3) in essence) the limiting eigenvalue distribution of these random matrices with $H_{0}=0$ is again the semicircle law if $\nu=0$. The case of $0<\nu<1$ is more complicated (for details see [4, 17]).

We have mentioned in the Introduction that there exists an analogy between the spectral problems which we are studying in this paper and the mean field theories in statistical mechanics and solid state theory. Therefore it is natural to compare our results with results of mean field type approximations developed in the theory of disordered systems (see reviews [5, 21,34]). These approximations are known also as single-site approximations and are applied mainly to the averaged Green function of the respective random operator.

The most widely accepted approximation of this type is the coherent potential approximation (CPA). It was proposed and applied to random operators with a diagonal disorder, i.e. the discrete Schrödinger operator

$$
-h \Delta_{\text {disc }}+q(x), \quad x \in \mathbb{Z}^{d}
$$

with an i.i.d. random potential first of all. Therefore one cannot expect too much similarity between our results obtained for the "opposite" case of the "maximum" off-diagonal disorder and results of the CPA. In particular for the Cauchy-distributed random potential (the Lloyd model), when

$$
P\{q(x) \in d q\}=\gamma \pi^{-1}\left(q^{2}+\gamma^{2}\right)^{-1} d q, \quad \gamma>0,
$$

the basic relations of the CPA $[8,21,34]$

$$
\begin{gathered}
\mathbb{E}\{G(x, x ; z)\}=G_{0}(0, z+\Delta(z)), \\
\mathbb{E}\left\{\frac{q(x)+\Delta(z)}{1+[q(x)+\Delta(z)] G_{0}(0, z+\Delta(z))}\right\}=0
\end{gathered}
$$

yield the following for the selfenergy $\Delta(z)$,

$$
\Delta(\lambda+i \varepsilon)=i \gamma \operatorname{sign} \varepsilon .
$$


Since according to our notation the 1.h.s. of (7.3) is $r(z)$, we can write (7.3) and (7.5) for $\operatorname{Im} z \neq 0$ as

$$
r(z)=r_{0}(z+i \gamma)
$$

This formula is obviously different from our basic equation (2.15) which determines the selfenergy

$$
\Delta(z)=w^{2} r(z)
$$

as the solution of this equation.

Nevertheless, as was shown in [31], Eqs. (7.3) and (7.4) yield (2.15) if the probability distribution of $q(x)$ is the semicircle law (5.6).

According to many suggestions and numerical results, the accuracy of the CPA increases with the increase of the coordination number of a lattice, i.e. its dimensionality in particular (note that no general quantitative criteria for the validity of the CPA seem to be available). Consider in this connection the Lloyd model (7.1), (7.2) again. It is well known that for this model [21]

$$
\mathbb{E}\{G(x, y ; \lambda+i \varepsilon)\}=G_{0}(x-y ; \lambda+i \varepsilon+i \gamma \cdot \operatorname{sign} \varepsilon) .
$$

Comparing this relation with (7.3) and (7.5) we conclude that the CPA is exact for the Lloyd model. Now, rescaling the translation invariant part of (7.1) in accordance with (2.2b):

$$
h=h_{d} d^{-1 / 2},
$$

we find from (7.8) with $x=y$ and $\operatorname{Im} z>0$ that

$$
r(z) \equiv \lim _{d \rightarrow \infty} \mathbb{E}\{G(x, x ; z)\}=\int(\lambda-z)^{-1} N_{0}(d \lambda),
$$

where $N_{0}(d \lambda)$ is the Gaussian distribution given by (2.17). This formula as well as (7.6) is different from Eq. (2.15) (with the same $N_{0}(d \lambda)$ ) according to which the selfenergy is given by (7.7).

Thus, at least in the case of the Lloyd model, the $d=\infty$ limits of the exact averaged diagonal element of the Green function and of its CPA form are different from the same limit of $\mathbb{E}\{G(x, x ; z)\}$ for the random operator (2.2) with an off-diagonal disorder satisfying $(2.1 \mathrm{e}, \mathrm{f})$. This conclusion is supported by recent diagrammatic analysis [30], according to which the $d=\infty$ limit of $\mathbb{E}\{G(x, x ; z)\}$ and of its CPA form coincide for an arbitrary random i.i.d. potential in (7.1).

It is worth noticing, perhaps as a kind of curiosity, that in the case of the Bethe lattice the infinite coordination number limit yields the deformed semicircle law, i.e. Eq. (2.15), even for the diagonal disorder model. Indeed, let us consider the discrete Schrödinger operator with an i.i.d. random potential on the Bethe lattice with the connectivity $K(K-1$ is the number of neighbours to a given site and plays the role of the coordination number of the dimensionality for the Bethe lattice). If $G_{i j}(z)$ is the respective Green function and $\Delta_{j}(z)$ is defined as

$$
\left[G_{\jmath j}(z)\right]^{-1}=q_{i}-z-\Delta_{\imath}(z)
$$

then according to [1]

$$
\Delta_{i}(z)=h^{2} \sum_{j}\left[q_{j}-z-\Delta_{j}(z)\right]^{-1}
$$


where sum in the r.h.s. is over $K$ nearest neighbours of a site $i$. Due to the special structure of the Bethe lattice (any branch of a given generation can be decomposed into $K$ identical and nonintersecting branches of the next generation), the $K$ random variables in the r.h.s. of (7.11) are all identically distributed and independent of one another and of the $K$ random i.i.d. variables $q_{j}$. These $2 K$ random variables generate the probability distribution of $\Delta_{i}$ in the l.h.s. which coincide with the distribution of each $\Delta_{j}$ in the r.h.s. of (7.11). Our intention is to consider the limit $K \rightarrow \infty$ for this recurrent relation. To this end we rescale the hopping integral $h$ as follows: $h=w K^{-1 / 2}$. Since $K$ plays the role of the dimensionality here, this scaling is analogous to the scaling $d^{-1 / 2}$ in (2.2b) and (7.9) in the translationally invariant part of (2.2) and (7.1). Using this rescaling it is easy to prove that the variance of the r.h.s. of (7.11) is $O\left(K^{-1}\right)$. Thus, each $\Delta_{i}$ converges in probability to a nonrandom limit as $K \rightarrow \infty$. Denoting this limit as $\Delta(z)$ and using (7.10) and (7.11), we obtain the relations

$$
\begin{gathered}
r(z) \equiv \mathbb{E}\left\{G_{\imath \imath}(z)\right\}=\int(q-z-\Delta(z))^{-1} F(d q), \\
\Delta(z)=w^{2} \int[q-z-\Delta(z)]^{-1} F(d q),
\end{gathered}
$$

where $F(d q)$ is the probability distribution of the potential $q_{i}$. Thus, $\Delta(z)$ satisfies (7.7), and as a result we obtain Eq. (2.15) determining the deformed semicircle law in which the role of an unperturbed IDS $N_{0}(d \lambda)$ is played by the probability distribution of the diagonal part of our random operator and the role of the variance $w^{2}$ of off-diagonal entries [see (2.1f)] is played by the squared rescaled hopping integral. Comparing this result with the respective results for the ensembles (2.2) and (5.4), we conclude that for the Bethe lattice the infinite coordination number limit yields the deformed semicircle law for the IDS even in the case of pure diagonal disorder and a properly scaled nonrandom off-diagonal part while, for a real lattice this eigenvalue distribution seems to be the case only for a considerable amount of off-diagonal disorder with the same scaling.

The above situation should also be contrasted with statistical mechanics where the Curie-Weiss formulae can be obtained as the infinite dimensionality limit of the respective formulae for the real lattice $\mathbb{Z}^{d}$ and as the infinite $K$ limit for the Bethe lattice (see e.g. [14]), while for the Schrödinger equation with a random potential these two limits are different: the former one results in the CPA and the latter one results in the deformed semicircle law.

To this point we have discussed the first moment of the Green function which determines equilibrium characteristics of disordered systems. However, to calculate kinetic characteristics, the conductivity first of all, we need to know the second moment of the Green function. It was recognized long ago $[8,34]$ that the simple decoupling $\mathbb{E}\{G G\}=\mathbb{E}\{G\} \mathbb{E}\{G\}$, which is in the spirit of any single-site (mean field type) approximation including the CPA is inconsistent with certain physical and mathematical conditions. The most important is the Hilbert identity which plays the role of the Ward identity here (see Remark 3 in Sect. 3). It was also found that a modification of this decoupling which is free of the above mentioned inconsistencies should only take into account the multiple scattering of two particles by the same site [29].

As we have mentioned above the infinite $R, d$ and $n$ limits do not coincide with the CPA. However these limits have similar properties. In particular, according to 
Theorem 4.1, all the Green function moments decouple into the products of the first moments in these limits and, according to Sect. 6, this fact does not contradict the Hilbert identity. The reason is not too fast decay of the respective remainders. As a result we cannot perform these limits inside the sums over the whole lattice entering into the Hilbert identity or in expressions (6.6), (6.29) and (6.39) for the Stieltjes transform of the conductivity. According to Sect. 6 [see the last part of this section starting from (6.34)], to calculate an expectation containing the sums mentioned we are to consider a larger family of quantities including both the products of the Green functions and certain infinite sums of some of these products and to derive a new infinite system of equations for the expectations of this family. However the common feature of this new system and the systems for the expectation of the products of the Green functions is that in both cases the solutions of the respective limiting systems have a factorized form [see (4.2), (6.24) and (6.41)] in accordance with the spirit of the single-site approximations.

This should be regarded as the proper mathematical mechanism of these approximations.

It is worth noting that, though the respective corrections (see e.g. (6.27)) are necessary to guarantee the Hilbert identity, they do not contribute to the conductivity (6.6) due to the antisymmetry of the velocity operator $\hat{\nu}$ in (6.5). Thus, we can use the simple decoupling $\mathbb{E}\{G G\}=\mathbb{E}\{G\} \mathbb{E}\{G\}$ in calculation of the conductivity. This fact is also well known in the theory of disordered systems (see $[8,21,34])$.

Vanishing of the simplest two-body corrections to the conductivity seems physically related to vanishing of the backscattering term in a transport equation [29]. As is generally accepted (see e.g. review [19]), the absence of the backscattering contribution is in turn closely related to the absence of localization. This is in agreement with the absence in our formulae of such well known manifestations of localization as exponential tails of the IDS, vanishing of the zero frequency conductivity for the Fermi energies for which the density of states is nonzero, etc.

Since for finite $R, d$, and $n$ the localized states should always be present at least at the edges of the spectrum of the operators (2.1)-(2.3), we have to conclude that the limiting transitions $R, d, n \rightarrow \infty$ "remove" completely the pure point spectrum of these operators. In particular, since according to (2.18) and (2.29) in all three limits (as well as in the CPA) the support of the zero-frequency conductivity as a function of the Fermi energy coincides with the IDS support, then one may speculate that the edges of this support coincide with the $R, d, n \rightarrow \infty$ limits of the mobility edges of respective operators.

\section{Appendix}

Let us show that the $R=\infty$ limit of the IDS (2.4) is independent of the diagonal random entries $W(x, x), x \in \mathbb{Z}^{d}$.

It suffices to prove that this property holds for $\lim _{R \rightarrow \infty} \mathbb{E}\left\{G_{R}(0,0)\right\}, \operatorname{Im} z \neq 0$. Assume now that in $(2.1) W(x, x)$ are arbitrary i.i.d. random variables such that

$$
\mathbb{E}\{W(x, x)\}=0, \quad \mathbb{E}\left\{W^{2}(x, x)\right\}=w_{1}^{2} \geq 0 .
$$


In other words, we replace (2.1e) by the most general form

$$
\begin{aligned}
\mathbb{E}\left\{W\left(x_{1}, y_{1}\right) W\left(x_{2}, y_{2}\right)\right\}= & w^{2}\left[\delta\left(x_{1}-x_{2}\right) \delta\left(y_{1}-y_{2}\right)\right. \\
& \left.+\delta\left(x_{1}-y_{2}\right) \delta\left(y_{1}-x_{2}\right)\right]\left[1-\delta\left(x_{1}-y_{1}\right)\right] \\
& +w_{1}^{2} \delta\left(x_{1}-x_{2}\right) \delta\left(y_{1}-y_{2}\right) \delta\left(x_{1}-y_{1}\right) .
\end{aligned}
$$

Denote by $\hat{H}_{R}$ the operator (2.1) in which all $W(x, x), x \in \mathbb{Z}^{d}$, are set to be zero and by $\hat{G}_{R}$ the Green function of $\hat{H}_{R}$.

Then the resolvent identity (3.8) with $A+B=H_{R}, A=\hat{H}_{R}$ yields

$$
\begin{gathered}
\mathbb{E}\left\{\left|G_{R}(0,0)-\hat{G}_{R}(0,0)\right|\right\} \leq \phi(0) R^{-d / 2} \sum_{s} \mathbb{E}\left\{\left|G_{R}(0, s) W(s, s) \hat{G}_{R}(s, 0)\right|\right\} \\
\leq \phi(0) R^{-d / 2}\left[\mathbb{E}\left\{\sum_{s}\left|G_{R}(0, s)\right|^{2}\right\} \mathbb{E}\left\{\sum_{s}\left|\hat{G}_{R}(0, s)\right|^{2} W^{2}(s, s)\right\}\right]^{1 / 2} .
\end{gathered}
$$

According to (3.11) the first expectation here is bounded from above by $\eta^{-2}$, $\eta=|\operatorname{Im} z|$. The second expectation is

$$
\sum_{s} \mathbb{E}\left\{\left|\hat{G}_{R}(0, s)\right|^{2}\right\} \mathbb{E}\left\{W^{2}(s, s)\right\}=w_{1}^{2} \mathbb{E}\left\{\sum_{s}\left|\hat{G}_{R}(0, s)\right|^{2}\right\}
$$

because $\hat{G}_{R}$ is independent of $W(x, x)$. By using once again (3.11), we arrive at the bound

$$
\mathbb{E}\left\{\left|G_{R}(0,0)-\hat{G}_{R}(0,0)\right|\right\} \leq \phi(0) w_{1}^{2} R^{-1 / 2} \eta^{-2} .
$$

Similar bounds are also valid for $a=d, n$.

Acknowledgements. L.P. is grateful to Prof. A.-M. Boutet de Monvel for kind hospitality at University Paris VII where this paper was partially written.

\section{References}

1. Abou-Chacra, R., Anderson, P., Thouless, D.: A selfconsistent theory of localization. J. Phys. C6, 1734-1752 (1973)

2. Akhiezer N.: The classical moment problem. London: Oliver and Boyd 1964

3. Berlin, T., Kac, M.: The spherical model of a ferromagnet. Phys. Rev. 86, 821-825 (1952)

4. Bogachev, L., Molchanov, S., Pastur, L.: On the density of states of random band matrices (in Russian). Mat. Zametki, 50, 31-42 (1991)

5. Brezin, E., Itzykson, C., Parisi, G., Zuber, J.: Planar diagrams. Commun. Math. Phys. 59, 35-51 (1978)

6. Casati, G., Molinari, L., Izrailev, F.: Scaling properties of band random matrices. Phys. Rev. Lett. 64, 1851-1854 (1990)

7. Constantinescu, F., Felder, C., Gawedzki, K., Kupiainen, A.: Analyticity of density of states in a gauge invariant model of disordered systems. J. Stat. Phys. 48, 365-391 (1987)

8. Elliot, P., Krumhansl, J., Leath, P.: Theory and properties of randomly disordered crystals and related physical systems. Rev. Mod. Phys. 46, 463-510 (1974)

9. Fernandez, R., Fröhlich, J., Sokal, A.: Random walks, random surfaces, critical phenomena and triviality in quantum field theory. Berlin, Heidelberg, New York: Springer, 1992

10. Girko, V.: Spectral theory of random matrices (in Russian). Moscow: Nauka 1988

11. Haake, F.: Quantum signatures of chaos. Berlin, Heidelberg, New York: Springer 1991 
12. Kac, M.: Mathematical mechanisms of the phase transitions. In: Chretien, M., Deser, S. (eds.): Statistical physics, phase transitions and superfluidity, Vol. I, pp. 241-301. New York: Gordon and Breach 1968

13. Kato, T.: Perturbation theory for linear operators. Berlin, Heidelberg, New York: Springer 1966

14. Kubo, R.: Statistical mechanics. Amsterdam, North-Holland 1965

15. Kac, M., Thompson, C.: Spherical model and the infinite spin dimensionality limit. Physica Norwegica 5, 163-168 (1971)

16. Khorunzhy, A., Pastur, L.: On the eigenvalue distribution of the deformed Wigner ensemble of random matrices. In: Operator theory and related topics. AMS (in press)

17. Khorunzhy, A., Molchanov, S., Pastur, L.: On the eigenvalue distribution of band random matrices in the limit of their infinite order (in Russian). Teor. Mat. Fiz. 90, 163-178 (1992)

18. Khorunzhy, A., Khoruzhenko, B., Pastur, L., Shcherbina, M.: The large- $n$ limit in statistical mechanics and the spectral theory of disordered systems. In: Domb, C., Lebowitz, J. (eds.): Phase transitions and critical phenomena Vol. 15, pp. 73-239. New York: Academic Press 1992

19. Lee, P., Ramakrishnan, T.: Disordered electronic systems. Rev. Mod. Phys 57, 287-337 (1985)

20. Lebowitz, J., Penrose, 0.: Rigorous treatment of the van der Waals-Maxwell theory of the liquidvapour transition. J. Math. Phys 7, 98-110 (1966)

21. Lifshitz, I., Gredeskul, S., Pastur, L.: Introduction in the theory of disordered systems. New York: Wiley 1988

22. Mehta, M.: Random matrices. New York: Academic Press 1967

23. Pastur, L.: Spectra of random self-adjoint operators. Russ. Math. Surv. 28, 1-67 (1973)

24. Pastur, L.: On the spectrum of random matrices (in Russian). Teor. Mat. Fiz. 10, 102-112 (1973)

25. Pastur, L., Figotin, A.: Spectra of random and almost periodic operators. Berlin, Heidelberg, New York: Springer 1992

26. Pastur, L., Shcherbina, M.: Infinite correlation radius limit for correlation functions of lattice systems (in Russian). Teor. Mat. Fiz. 61, 3-16 (1984)

27. Shcherbina, M.: Spherical limit of $n$-vector correlations (in Russian). Teor. Mat. Fiz. 77, 460-471 (1988)

28. Stanley, H.: Spherical model as a limit spin dimensionality. Phys. Rev. 176, 718-721 (1968)

29. Velicky, B.: Theory of electronic transport in disordered binary alloys: coherent potential approximation. Phys. Rev. 184, 614-627 (1969)

30. Vlaming, R., Vollhardt, D.: Controlled mean field theory for disordered electronic systems: single particle properties. Rutgers preprint RWTH/ITP - C 6/91

31. Wegner, F.: Disordered system with $n$ orbitals per site: $n=\infty$ limit. Phys. Rev. B19, 783-792 (1979)

32. Wigner, E.: Random matrices in physics. SIAM Review J. 9, 1-23 (1967)

33. Wegner, F., Opperman, R.: Disordered systems with $n$ orbitals per site: $1 / n$ expansion. Z. Phys. B 34, 327-348 (1979)

34. Yonezawa, F., Morigaki, K.: Coherent potential approximation. Suppl. Progr. Theor. Phys. 53, $1-76(1973)$ 